\title{
Experimental investigation of shock-induced separation and flow control in a transonic compressor cascade
}

\author{
Joachim Klinner ${ }^{1}$ (I) $\cdot$ Alexander Hergt ${ }^{2} \cdot$ Sebastian Grund ${ }^{2} \cdot$ Christian E. Willert $^{1}$
}

Received: 17 September 2018 / Revised: 14 April 2019 / Accepted: 23 April 2019

(c) Springer-Verlag GmbH Germany, part of Springer Nature 2019

\begin{abstract}
The influence of transition control on shock-induced flow separation was investigated in a highly loaded transonic compressor cascade at an inlet Mach number of 1.21 and a chord-based Reynolds number of $1.4 \times 10^{6}$. Transition was influenced by raising the free-stream turbulence from 0.5 to $2.5 \%$. Two further cases employed either air-jet vortex generators (AJVG) or a surface roughness patch as transition control devices. Velocity fields in the vicinity of the unsteady transonic separation were captured by particle image velocimetry (PIV). Blade flexure induced by the unsteady aerodynamic loading was tracked for each image and compensated individually prior to PIV processing. The captured flow fields indicate shape variations of the separation region, while the shock foot moves within a range of up to $20 \%$ of chord. The frequency of separation for each investigated case was assessed on the number of vectors with negative velocity in each PIV sample. To further quantify the size of the separation region, the statistically independent PIV samples were conditionally averaged for various passage shock positions at a resolution of $1 \%$ chord length. Insight to the dynamics and frequency of the passage shock motion was further provided by high-speed shadowgraphy. Large bubble separation occurs if the turbulence of the incoming flow is low. The size of separation region decreases when AJVGs are applied but still exhibits bubble separation as the passage shock moves downstream. The size of the separation region is significantly reduced either if a roughness patch is applied or if the turbulence level of the incoming flow is high. The flow conditions showing bubble separation in the mean flow also exhibit distinct spectral peaks indicating periodic shock oscillations.
\end{abstract}

Electronic supplementary material The online version of this article (https://doi.org/10.1007/s00348-019-2736-z) contains supplementary material, which is available to authorized users.

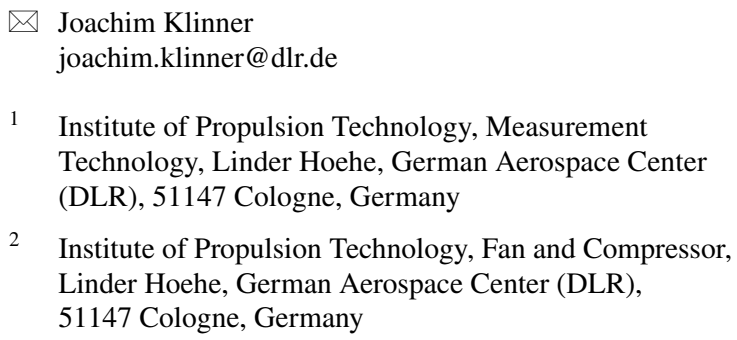

2 Institute of Propulsion Technology, Fan and Compressor, Linder Hoehe, German Aerospace Center (DLR), 


\section{Graphical abstract}

Separation control devices

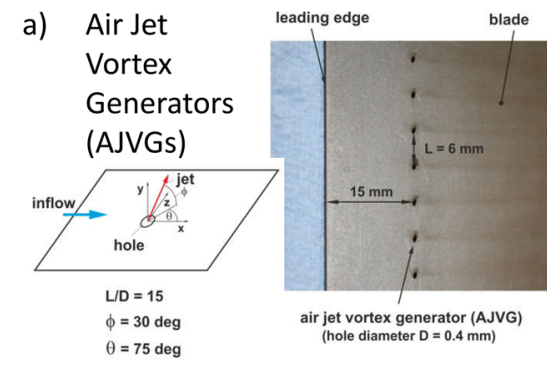

b) Surface Roughness Patch (SRP)

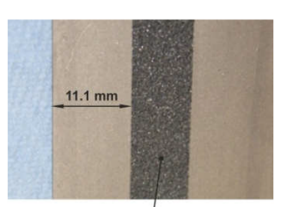
roughness patch
$(\mathrm{Ra}=10 \mu \mathrm{m}$, width $=10 \mathrm{~mm})$
Cascade wind tunnel

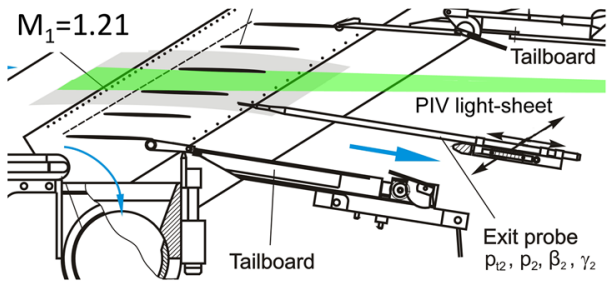

Cascade shock system

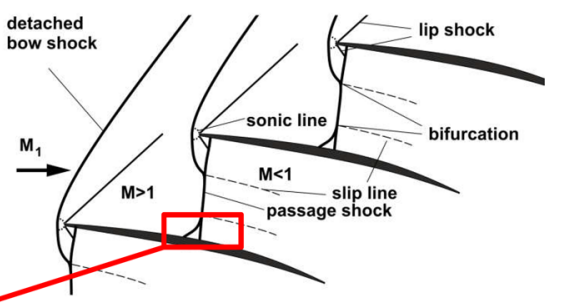

PIV of the unsteady shock BL Interaction

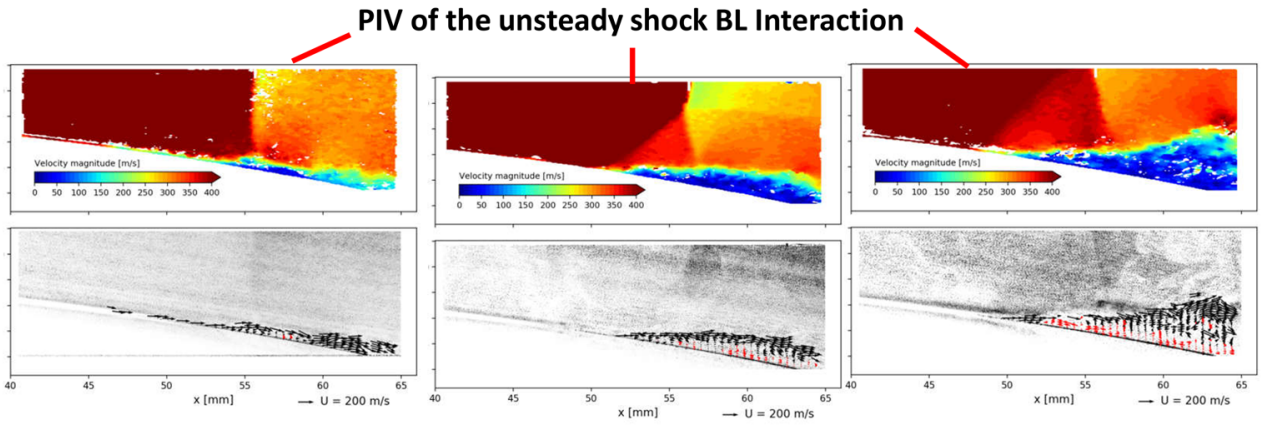

Comparison of effectivity of separation control

Frequency of separation

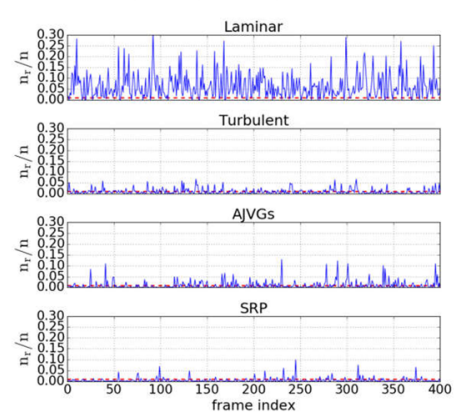

Position and size of the separation region

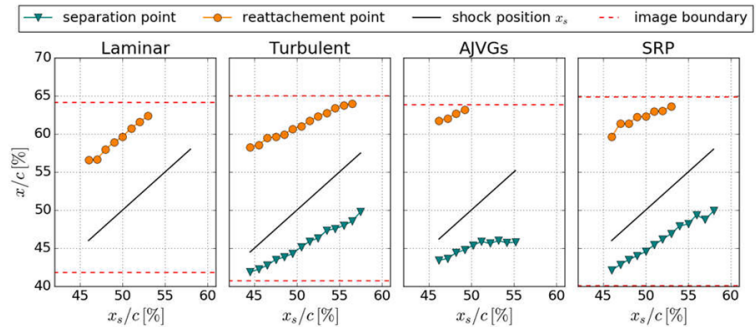

\section{Introduction}

The design of transonic blades for aero engine fans or axial compressors requires a correct estimation of the shock wave-boundary layer interaction (SBLI) phenomenon. The interaction is often coupled with large amplitudes and possibly self-sustained oscillations of normal or near-normal shocks both of which are highly undesirable as they lead to unsteady aerodynamic and structural loading (Lee 2001; Dussauge and Piponniau 2008). Especially, low-frequency motion of the normal shock is accompanied by low-frequency pressure fluctuations on the blade surface which, when on the order of structural modes, in turn reduce the buffet boundary or even further increase the magnitude of the blade vibrations. As a consequence, machine maintenance cycles are shortened and possibly alongside with an overall reduction of component/machine life time. Beyond this, there is also the fact that the associated flow separation additionally enhances the viscous losses of the compressor blading by strongly enhancing dissipation in the boundary 
layer (BL) (Délery and Bur 2000). Also, the formation of large-scale eddies in the separation region may impose unsteady downstream conditions to the shock which can be a further source of large-scale shock unsteadiness (Dussauge and Piponniau 2008).

Although not so common in the field of turbomachinery, considerable research already has been conducted in an effort to understand and to control shock-induced flow separation and shock oscillations on airfoils (Délery and Marvin 1986; Dolling 2001; Dussauge and Piponniau 2008; Giannelis et al. 2017). Despite the fact that a robust prediction of the buffet boundaries and a comprehensive explanation of the underlying mechanisms of shock oscillations are still not available (Giannelis et al. 2017), the recent literature on this topic provides some results from experimental investigations. Sartor et al. (2012) studied the transonic interaction between a strong steady shock wave and a turbulent BL downstream of a bump at $M a_{1}=1.4$. Their results provide a detailed description of the lambda-shaped shock system along with velocity fluctuations in the separation region. The authors conclude that the separated flow exhibits both strong anisotropy and a high degree of turbulent kinetic energy in the shear layer, which in turn promote the presence of large-scale vortices. Another detailed experimental study on transonic buffeting over a supercritical wing profile was conducted by Jacquin et al. (2009) using oil film visualizations, time-resolved pressure measurements and two-component LDV at several chord-wise stations. The authors applied phase-averaging of LDV data based on the dominant buffet frequency. The latter was obtained from simultaneous Kulite pressure measurements performed near the mean shock foot position. Results show that both shock motion and separation are closely coupled and exhibit a mainly chord-wise periodic behaviour. The dynamic behaviour of shock-induced flow separations above a supercritical airfoil was investigated by Hartmann et al. (2012, 2013). Here, the authors applied high-speed stereoscopic PIV and Kulite measurements near the shock-induced separation region and provided experimental evidence that a dominant mechanism for self-sustained shock oscillations is a feedback loop between shock position and upstream traveling sound waves that originate from the trailing edge as described by Lee (2001). Also, they showed that shock oscillations can be influenced by acoustic excitation with an external source.

The major objective of the present research is to study the effectiveness of separation control in a highly loaded transonic axial compressor cascade with regard to reduction of shock-induced separation in size and unsteadiness. The aim is to assess to what extent the size of the flow separation is reduced, while the momentum of the BL upstream of the SBLI is increased. One method to achieve momentum exchange between the outer high-speed flow and the BL is distributed fluid injection that generates streamwise vortical structures (Délery and Bur 2000). Aimed at the application in turbomachinery, separation control by passive air-jet vortex generators (AJVGs) was recently investigated by Szwaba $(2011,2013)$ and Tejero et al. (2015). Another approach to separation control is to trigger the laminar-turbulent transition upstream of the SBLI to increase the momentum in the upstream BL profile making it less prone to separation. Parametrization of transition control by distributed roughness in a single transonic compressor passage was also recently investigated by Szwaba et al. (2019).

In the present research, specific configurations of AJVGs and of distributed roughness are applied to an existing compressor blading geometry in a cascade wind tunnel. Frequency, size and position of the unsteady shock-induced separation of different types of flow control are assessed by liquid crystal visualizations, classical two-component (2C) PIV and on the basis of conditional averaging over the shock position using large sets of PIV samples. The present investigation sacrifices time resolution [such as provided by high-speed PIV investigations of SBLIs by Hartmann et al. (2012)] in favor of a fourfold increase in spatial resolution in the region covering the shock foot (e.g. lambda pattern) and the associated separation region. The increased spatial resolution allows an improved resolution of the complex shock patterns as well as vortices in the separation region and slip surfaces emanating from bifurcation points of the shock pattern. In addition to the assessment of the size of the separation region, harmonic components of shock buffet are recovered through Fourier analysis of shock positions obtained from time-resolved shadowgraph imaging.

\section{Experimental methods}

\subsection{Test facility and configurations under investigation}

Measurements investigating the aforementioned influences of transition control were conducted in the transonic cascade wind tunnel of the DLR in Köln (see Fig. 1) at $M a_{1}=1.21$. The tunnel is a closed-loop facility capable of continuous operation. The incoming flow passes through a straight cascade of six airfoils representing a portion of a ring of an actual blade row in an axial compressor or fan. The cascade blades are supported by side walls made of acrylic glass which at the same time provide suitable optical access. BL suction capacities of the facility allow the control of the side wall BL in front of the cascade test section. By controlling the latter, a homogeneous in-flow representative of an "infinite blade cascade" can be achieved. In addition, suction slots on the end walls inbetween the blades provide control 
(a)

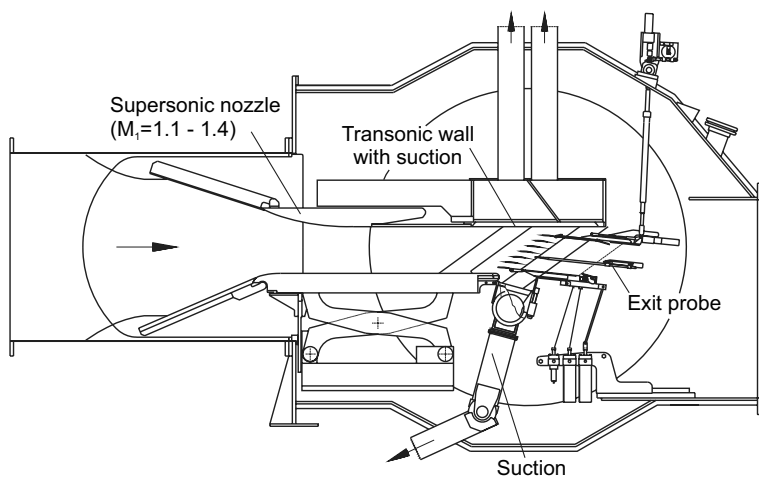

(b)

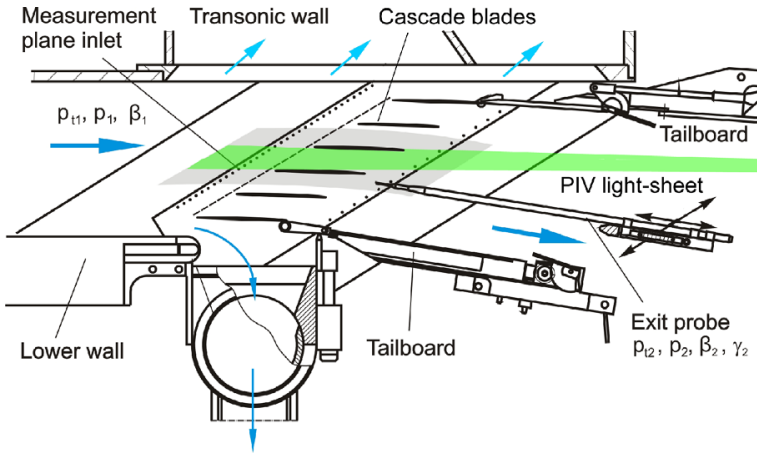

Fig. 1 a Schematic of the transonic cascade wind tunnel at the DLR Institute of Propulsion Technology; $\mathbf{b}$ test section and light sheet orientation for PIV

of boundary layers on the side walls within the cascade passages to adjust specific axial velocity density ratios at a controlled constriction (Hergt et al. 2016).

The present blade geometry was provided by Rolls-Royce Germany within the EU research project TFAST (transition location effect on shock wave boundary layer interaction). The cascade consists of six blades with $c=100 \mathrm{~mm}$ chord length and $168 \mathrm{~mm}$ blade span. At the aerodynamic design point, the blade system exhibits a flow turning of $15^{\circ}$. For the given inlet Mach number of $M a=1.21$, the chord Reynolds number is $1.4 \times 10^{6}$. The stagnation pressure varies between 90 and $95 \mathrm{kPa}$ and the stagnation temperature ranges between 300 and $305 \mathrm{~K}$. A detailed description of the blade geometry and operating conditions can be found in Hergt et al. $(2018,2019)$.

Among the four cases studied, the reference case ('laminar case') contains no additional flow conditioning by means of separation control or increase of turbulence. In this case, upstream of the cascade, the in-flow exhibits a low turbulence intensity ( $\mathrm{Tu}=0.5-0.8 \%)$ and the shock interacts with a laminar BL. For the second case ('turbulent case'), the turbulence level of the incoming flow is increased by a turbulence grid placed upstream of the test section leading to a turbulence intensity of $\mathrm{Tu}=2.25-2.5 \%$ in front of the leading edge. This way, the transition onset is shifted upstream

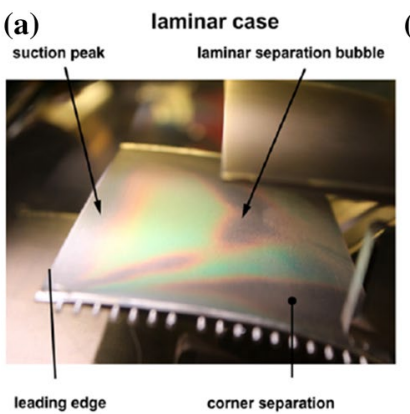

(b) laminar case + turbulator wedge

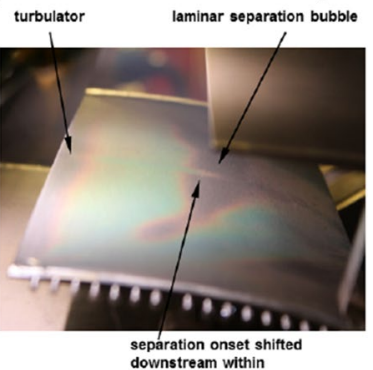

(c)

turbulent case downstream within
turbulator wake

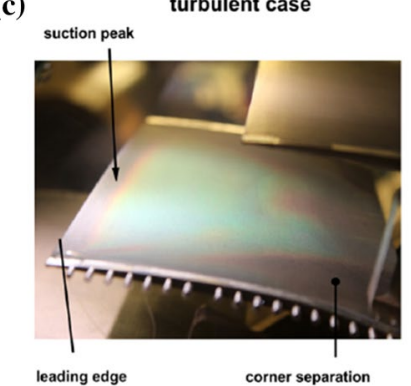

Fig. 2 Liquid crystal visualization on the central blade's suction side; a laminar BL upstream of the SBLI; b laminar BL with turbulator and with separation onset shifted downstream in the turbulator wake; c highly turbulent in-flow $(\mathrm{Tu}>2.25)$

of the normal shock with the shock wave interacting with a turbulent BL. The specified ranges of turbulence intensities were estimated from hot-wire measurements at subsonic velocity recorded at a distance of $60 \%$ of chord upstream of the cascade using an in-house hot-wire measurement technique (for details, see Meyer et al. 2010).

Laminarity of the BL upstream of the SBLI was visualized by a liquid crystal coating on the suction side of the central blade. During visualizations, the in-flow stagnation temperature is maintained to within $\pm 0.1 \mathrm{~K}$. The visible color range of the selected liquid crystals (R20C10W, LCR Hallcrest) is $10 \mathrm{~K}$ (for details see Hergt et al. 2018). For the visualization of the adiabatic wall temperature, the coating appears red for the lower temperature bound of $293 \mathrm{~K}$, which changes first yellow and then green with a temperature increase to $303 \mathrm{~K}$. Outside this range, the liquid crystals exhibit high absorption of visible light and thus appear black. Figure $2 \mathrm{a}, \mathrm{b}$ compares visualization results for the laminar case with and without a turbulator wedge that is placed near the leading edge. The dark region parallel to the leading edge is induced by the suction peak. A large separation region appears approximately at mid chord length. In the wake of the turbulator, the onset of the separation is shifted far downstream and the chord-wise extend of the separation region is reduced. This observation is considered as an indication of a laminar in-flow BL in the absence of the turbulator. For the turbulent in-flow condition (Fig. 2c), 
the separation region size is significantly smaller compared to the laminar test case.

With the intention of reducing the separation region, flow control was applied in two further cases. The first configuration made use of AJVGs that consist of a spanwise row of inclined bores of $0.4 \mathrm{~mm}$ diameter placed $15 \mathrm{~mm}$ $(x / c=15 \%)$ downstream of the leading edge as shown in Fig. 3a, b. Through these AJVGs, air coming from the pressure side is blown out in the form of inclined jets issuing into the airstream transverse to the main flow direction at the suction side. These jets are designed to produce streamwise vorticity, accompanied by momentum transfer from the outer high-speed flow to the BL, thus making it less prone to separation. Within the research project TFAST, geometry and position of these particular AJVGs were optimized both experimentally and numerically by the Szewalski Institute of Fluid-Flow Machinery of the Polish Academy of Sciences (IMP PAN) which provided a single blade test section using the same blade geometry and similar aerodynamic parameters as in the present cascade study (Szwaba 2013; Flaszynski et al. 2015; Tejero et al. 2015). The position with regard to the leading edge was optimized in such a way that the spacing between AJVGs and the mean shock position is as small as possible, while transition upstream of the SBLI is ensured.

In the second configuration, the laminar-turbulent transition is triggered at a fixed location upstream of the shock foot by a surface roughness patch (SRP) of $10 \mathrm{~mm}$ width that is placed $11.1 \mathrm{~mm}(x / c=11.1 \%)$ downstream of the leading edge as shown in Fig. 3c. The roughness parameters are $R_{\mathrm{a}}=10 \mu \mathrm{m}$ and $R_{\mathrm{z}}=35 \mu \mathrm{m}$. Again, position and size parameter as well as the optimum surface roughness parameters were previously optimized at the IMP PAN (Szwaba et al. 2019). The position of the SRP on the suction side was chosen such that transition is ensured upstream of the SBLI, while the extent of the turbulent BL upstream of the SBLI is kept as short as possible to avoid additional losses.

Static pressure taps, located at mid-span of the central airfoil of the cascade, allow the monitoring of the chord-wise static pressure and isentropic Mach-number distributions on the suction and pressure side. To establish comparable operating conditions for all configurations, flow conditions are set to match as much as possible a specific isentropic Machnumber distribution which corresponds to the aerodynamic design point of the blading. This chord-wise Mach-number distribution (see Fig. 4) confirms a coinciding shock position on the suction side for all configurations. Variations upstream of the steepest gradient $(x / c<0.5)$ on the suction side originate already from different $\mathrm{BL}$ conditioning (Hergt et al. 2018, 2019). In comparison to all other configurations,

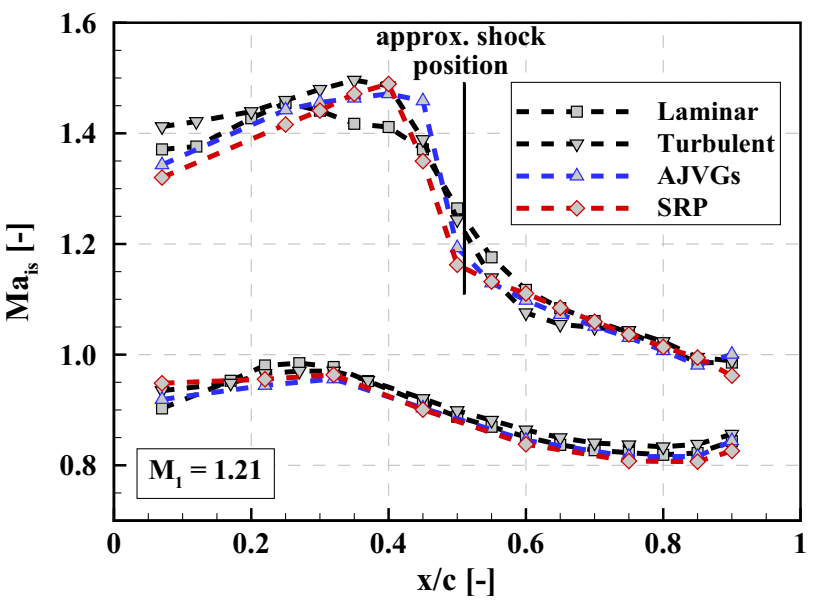

Fig. 4 Isentropic Mach-number distribution at mid-span (a)

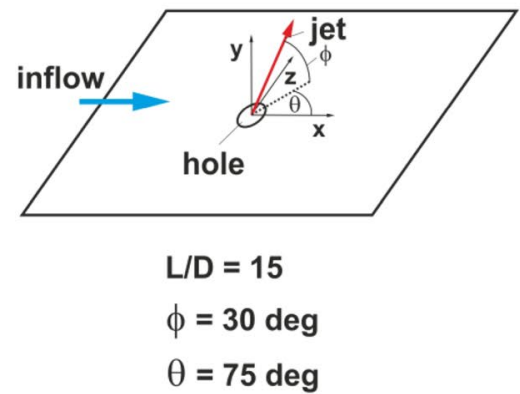

(b)

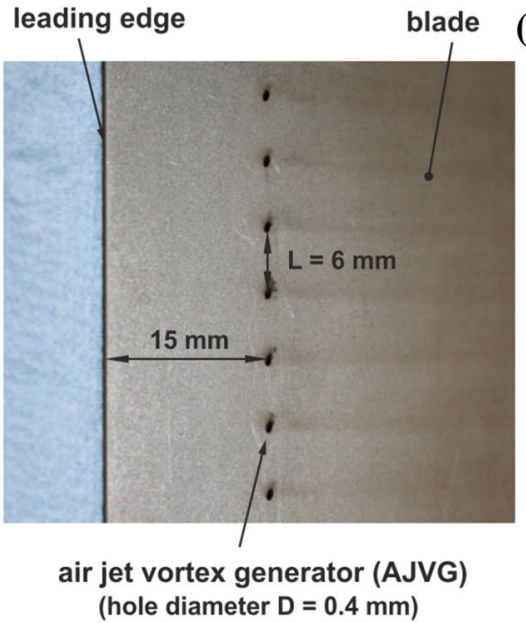

(c)

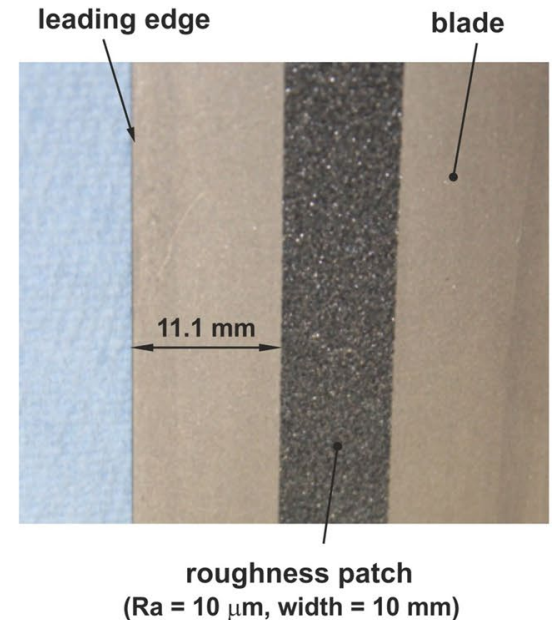

Fig. 3 a Schematic diagram of air-jet vortex generators (AJVGs); b AJVG arrangement on the suction side; $\mathbf{c}$ dimensions of the roughness patch on the suction side 
the laminar configuration shows the lowest pre-shock surface Mach number and the weakest gradient across the shock.

\subsection{PIV instrumentation and image processing}

A planar two-component PIV setup is applied using a classical normal viewing arrangement with the measurement plane located at mid-span. The double-frame camera (laminar case: PCO2000, other: ILA.SCMOS) is equipped with an Nikkor Micro $f=200 \mathrm{~mm}$ lens at a magnification of $M \approx 0.55$ or $10.0-11.2 \mu \mathrm{m} /$ pixel. The image size covering shock-foot and the associated separation region varies between $2048 \times 680$ pixels (laminar case) and $2560 \times 1024$ pixels (other). PIV measurements of the SBLI were conducted for a field that covers the shock foot and the separation region near the suction side (region B in Fig. 5b). Due to the optical access restrictions, the laser light is introduced through a light-sheet probe about $550 \mathrm{~mm}$ downstream from the cascade as illustrated in Fig. 1b. The far downstream distance of the light sheet probe is chosen to minimize disturbances of the flow in upstream direction. More details regarding the light sheet probe and seeding procedure are described by Klinner et al. (2014).

For measurements of the separation region, the tracer density of the conventional paraffine droplet seeding was found to be insufficient and thus, a smoke generator is used which generates tracer droplets by evaporation and re-condensation of refined mineral oil leading to droplet sizes considerably below $1 \mu \mathrm{m}$. The smoke was fed into the tunnel by a radial compressor pump and a seeding injection rake of $300 \times 300 \mathrm{~mm}^{2}$ which is installed within the settling chamber downstream of the diffusor screens about $3 \mathrm{~m}$ upstream of the test section. The pump also has the function of removing larger oil droplets from the seeding flow through high centrifugal forces. The tracer response to the normal shock was evaluated from PIV measurements in region A. For the evaluation of the response time, each single shot velocity profile is fitted against an exponential velocity profile (Ragni et al. 2011; Klinner et al. 2014). From this, the characteristic relaxation length of smoke oil tracer deceleration to the $1 / \mathrm{e}$ level of the velocity step across the normal shock is estimated with $\xi_{\mathrm{p}}=0.29 \pm 0.06 \mathrm{~mm}$ and an average relaxation time of $\tau_{\mathrm{p}}=0.94 \pm 0.18 \mu \mathrm{s}$. Laser energy of $25 \mathrm{~mJ}$ per pulse provides sufficient light scattering from the sub-micrometer particles by producing particle images with a signal of approximately $6 \%$ of the camera dynamic range. The maximum repetition rate of each double pulse is $15 \mathrm{~Hz}$ which currently limits the PIV sampling rate.

The unsteady aerodynamic loading of the thin blade results in a flexure of the blade, in particular at mid-span (first flexure mode) at the position of the PIV image plane defined by light sheet. In image space, the range of these vertical blade displacements is in the order of \pm 28 pixels $( \pm 0.27 \mathrm{~mm})$. Other displacements due to camera vibrations were found to be less than 1 pixel on the basis of recording a fixed point at the tunnel side wall. Using a correlation-based algorithm, the relative position of the blade surface was determined for each recording in a small rectangular region above the blade suction side as indicated in Fig. 6a. For this purpose, the region has been divided into strip-shaped interrogation windows. For each window, the first of both double frames is correlated with a reference frame recorded at a median blade position. Because PIV samples (each first frame) are recorded at 7.5-15 Hz, only the blade surface contributes to the correlation whereas the particle patterns are completely uncorrelated. Vertical displacement components of the blade surface are obtained for each interrogation window by a sub-pixel correlation peak detection. These vertical components are then validated and averaged to obtain a vertical blade displacements for each PIV sample that is used to offset the image data to a coincident blade position prior to further postprocessing of the recovered velocity data (see Fig. 6b). Due to the short pulse separation of $0.5 \mu \mathrm{s}$ between the laser pulses, blade motion within a single PIV recording could not be resolved. (a)

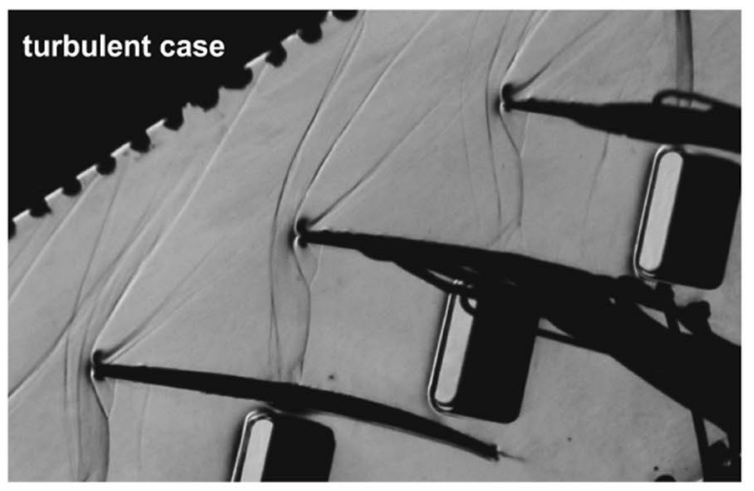

(b)

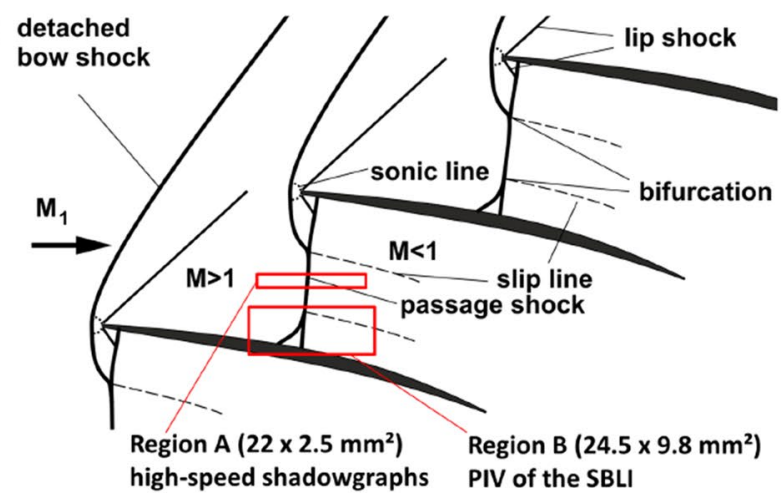

Fig. 5 a Schlieren image at turbulent conditions; $\mathbf{b}$ schematic diagram of the shock system including measurement regions 
(a)

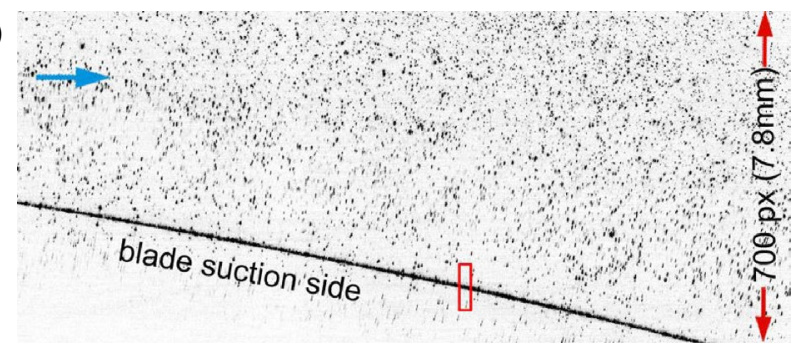

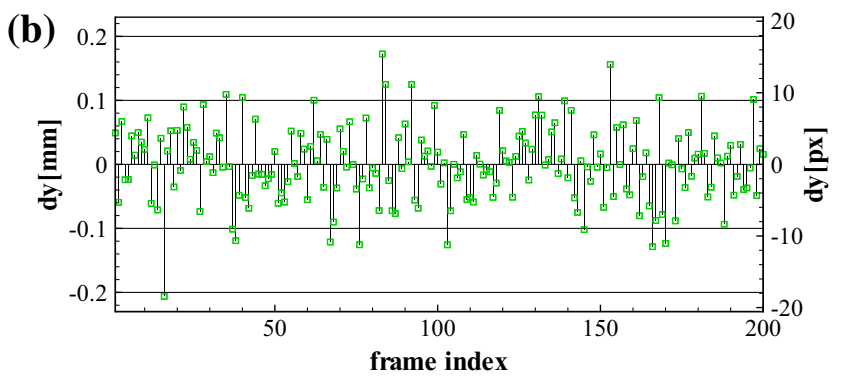

Fig. 6 a PIV raw image with rectangular region for evaluation of blade displacements; $\mathbf{b}$ vertical blade displacements over 200 frames measured within the red rectangular box

The PIV image data were processed using both in-house and commercial software (PIVview v3.8, PIVTEC GmbH) based on a coarse-to-fine multi-grid processing scheme with image deformation at each step (Scarano 2002) allowing to take into account the strong shear in the flow. The sub-pixel correlation peak position measurement was performed by a truncated sinc signal reconstruction algorithm. Due to this processing, an accuracy of correlation peak detection below 0.1 pixel can be obtained for regions without strong laser background and sufficient particle image density. Outliers were excluded based on a normalized median filter (threshold $=6$ ) and a dynamic mean test. An overview of achieved PIV resolutions and accuracies is provided in Table 1.

Under laminar conditions, the boundary layer upstream of the SBLI is practically devoid of particles. Immediately upstream of the SBLI, particles occasionally become visible in individual PIV images, which confirms an intermittent character of the BL which seems to switch between a laminar and turbulent state as already described in Giepman et al. (2015). This behaviour is illustrated in Fig. 7. Below and downstream of the SBLI, the particle image density increases rapidly through improved mixing with the outer flow. During PIV evaluation, outliers in isolated areas of low seeding density are filtered out based on a specific signal-to-noise ratio (SNR) of the signal peak. A vector is rejected if the SNR of the signal peak falls below 5 (white areas in Fig. 7).
PIV recordings with a inhomogeneous or weak seeding density have not been considered for statistical evaluation. A PIV sample is rejected if more than $10 \%$ of all visible vectors are outliers based on the above validation scheme.

Near the suction side, the shock-foot position was found to fluctuate within a range of up to $20 \%$ of chord $(20 \mathrm{~mm})$. To quantify the mean size of the separation region along with the position of the separation and reattachment points, conditional averaging was conducted based on the position of the shock foot in each PIV snapshot. To achieve a sufficient number of conditional samples, a total of at least 10,000 PIV samples were recorded per condition (cf. Table 1). After PIV processing and compensation of blade vibrations, the shock position in each sample was estimated through the maximum absolute axial velocity gradient $|\partial u / \partial x|$ across the normal shock. Therefore, velocities were averaged over seven top rows of the velocity field in region B (see Fig. 5b) and differentiation is conducted through a standard least squares approach over five nodes along $x$ (Raffel et al. 2017).

\subsection{High-speed shadowgraphy setup and processing}

To further analyze the passage shock oscillations with high spatial and temporal resolution, time-resolved shadowgraphs were recorded using pulsed LED back-illumination (Willert et al. 2012). The field of view covered a region of $24 \times 4.42 \mathrm{~mm}^{2}$ centered with respect to region

Table 1 PIV resolutions and accuracies for various test cases

\begin{tabular}{lllllll}
\hline & $\begin{array}{l}\text { Magnification }(\mu \mathrm{m} / \\
\text { pixel })\end{array}$ & $\begin{array}{l}\text { Pulse delay } \Delta t \\
(\mu \mathrm{s})\end{array}$ & $\begin{array}{l}\text { Field of view }(W \times H) \\
\left(\mathrm{mm}^{2}\right)\end{array}$ & $\begin{array}{l}\text { Inter. window } \\
(W \times H)\left(\mathrm{mm}^{2}\right)\end{array}$ & $\begin{array}{l}\text { Accuracy } \\
\varepsilon(\mathrm{m} / \mathrm{s})\end{array}$ & $\begin{array}{l}\text { Number of } \\
\text { PIV record- } \\
\text { ings }\end{array}$ \\
\hline Laminar case & 11.1 & 0.4 & $22.8 \times 7.8$ & $0.53 \times 0.27$ & 2.78 & 10,000 \\
Turbulent case & 9.56 & 0.5 & $24.4 \times 9.8$ & $0.46 \times 0.23$ & 1.91 & 1.96 \\
AJVG & 9.82 & 0.5 & $25.1 \times 10.1$ & $0.47 \times 0.2$ & 1.98 & 10,000 \\
SRP & 9.92 & 0.5 & $25.4 \times 10.2$ & $0.48 \times 0.2$ & 16,956 \\
\hline
\end{tabular}

${ }^{\mathrm{a}}$ Based on a positional detection accuracy of the signal peak of 0.1 pixel 
(a)
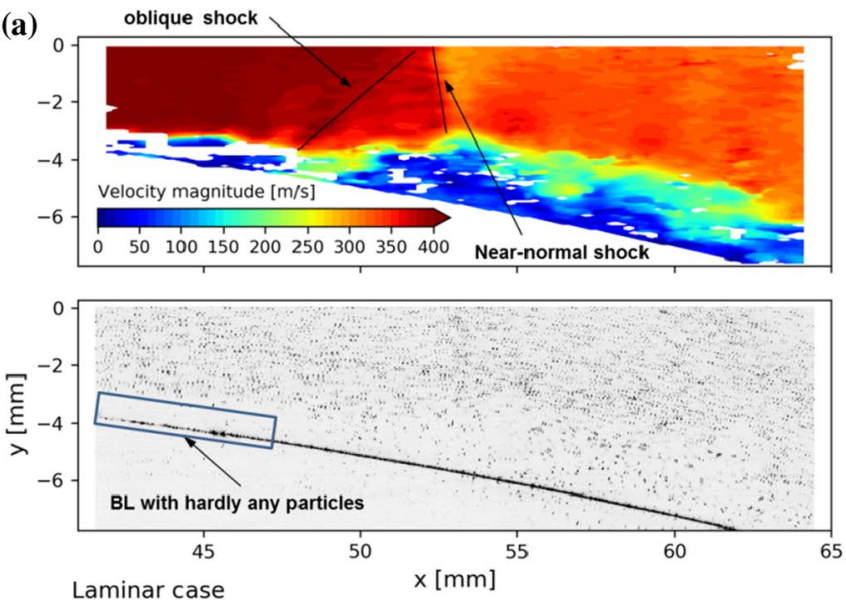

(b)
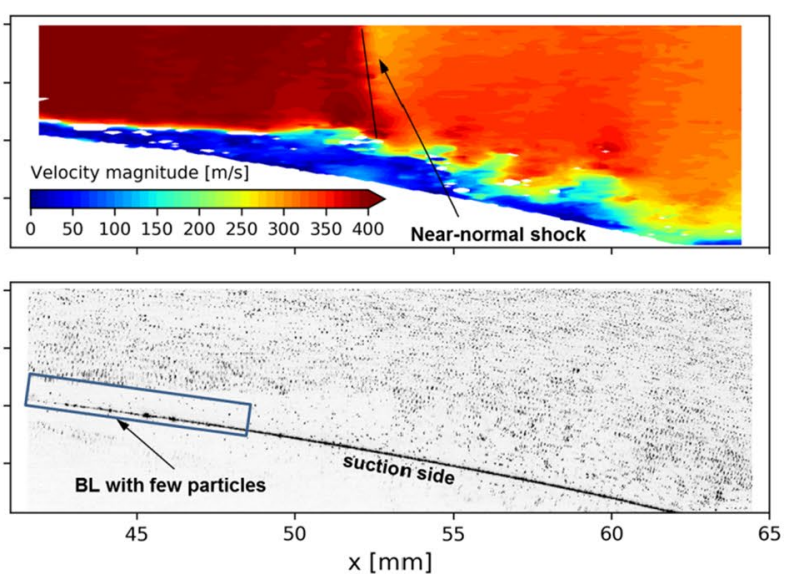

Fig. 7 Two PIV samples for laminar conditions with hardly any (a) or very few particles (b) in the BL immediately upstream of the SBLI; during PIV processing devoid regions have been filtered out based on a threshold of the signal-to-noise ratio (white contour)

A in Fig. 5b with the plane of focus positioned at midspan. The pulse duration was set to $0.4 \mu$ s with a repetition rate of $20 \mathrm{kHz}$ and synchronized with a high-speed camera (Photron SA-5) equipped with a $100 \mathrm{~mm}$ Nikkor Micro lens set at a magnification of $35 \mu \mathrm{m} /$ pixel. More details about the high-speed shadowgraphy system can be found in Klinner et al. (2014). For image processing, the shock-induced intensity gradients in each shadowgraph are enhanced by background subtraction and image normalization using the average intensity over each image sequence to obtain a homogeneous background intensity. The position of the passage shock $x_{\mathrm{s}}$ is tracked in each image based upon the maximum absolute intensity gradient (see
Fig. 8). Occasional erroneous detections due to the finite axial width of the shock shadowgraph were mitigated by a sliding median filter over three samples. The power spectral distribution of shock movement is obtained by a Fourier analysis of the shock position over time using image sequences of up to 127,000 samples. The processing is based on averaging the FFTs of a temporally sliding window of a fixed width and overlap originally introduced by Welch (1967). The herein presented data were spectrally analyzed using a sliding window of 2048 samples (102 ms) at $75 \%$ overlap. Furthermore, the window is weighted by a Hanning function to suppress processing artifacts. (a)

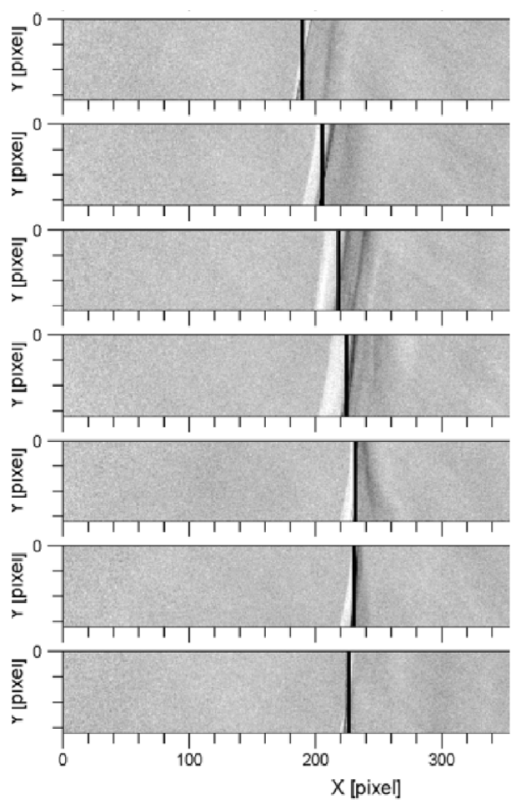

(b)

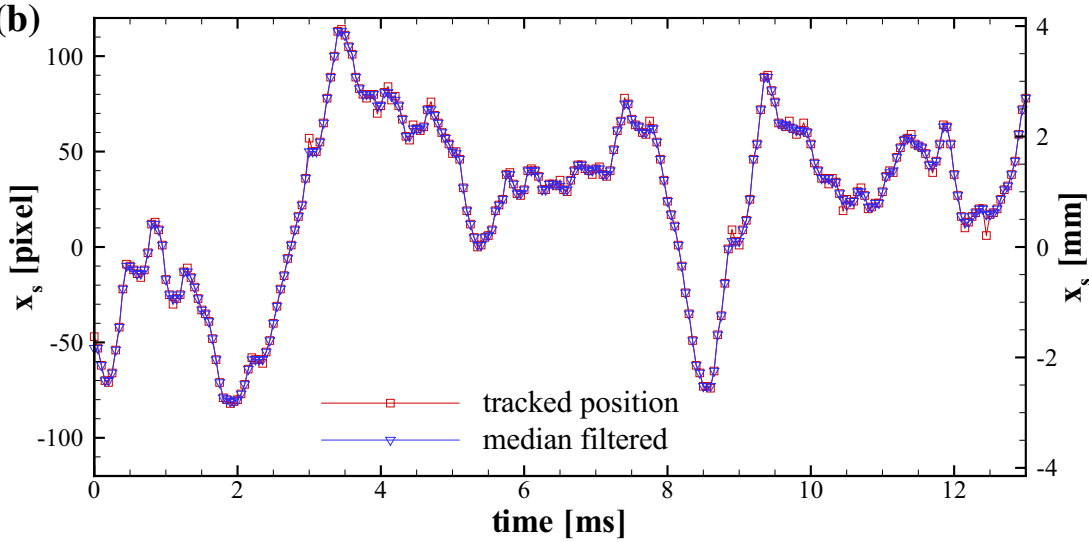

Fig. 8 Position of the passage shock over time in region A in Fig. 5b sampled at $20 \mathrm{kHz}$; a sequence of shadowgraphs; b shock position over time, erroneous detections due to the finite axial width of the shock shadowgraph were mitigated by a sliding median filter over three samples 


\section{Results and discussion}

\subsection{Unsteadiness of shock-boundary layer interaction}

For every case investigated, the interaction of shock waves and BL is highly unsteady which is illustrated with six PIV shots in Fig. 9 recorded for the turbulent test case. Herein, neither the passage shock position nor the extension of the separation region is steady. Independent of the position of the normal shock wave, different flow states can be identified. Some of the PIV recordings show a weak flow separation together with a normal shock wave with no or only a very weakly pronounced lambda shock system (see Fig. 9a). Other PIV samples exhibit a transonic separation including a lambda shock pattern that consists of a normal shock wave that splits into an oblique shock and a near-normal shock wave (see Fig. 9b). Here, the oblique shock starts from the point of separation, while the near-normal shock starts above the separation region. The flow within the shock structure is weakly transonic and becomes subsonic downstream of the near-normal shock. The reattachment point is shifted far downstream. Figure $9 \mathrm{c}$ shows partially occurring strong flow separations involving pronounced shape variations of the lambda pattern as well as large vortices occurring downstream of the rear shock.

Some of the PIV snapshots exhibit a subsonic flow downstream of the lambda pattern that is divided by a shear layer starting from the triple point (also known as bifurcation point), where normal shock, oblique shock and near normal shock intersect (see Fig. 10). In the literature, this shear layer is often referred to as 'slip line' or 'vortex sheet' (Délery and Marvin 1986; Babinsky and Harvey 2011). The slip line results from different increases in total entropy between the region downstream of the normal shock and the region downstream of the bifurcated oblique and nearnormal shock, leading to different post-shock velocities in both regions (Délery and Marvin 1986). The occurrence and position of the slip line vary widely with the shape of the separation region and the strength of the oblique shock, as indicated in Fig. 10. The initial velocity deficit downstream of the triple point is larger in Fig. 10a in comparison to Fig. 10b-d which is likely due to a lower total pressure loss in the lower shock structures also visible through different oblique shock angles. For the turbulent case, the slip line was visible only in about $7 \%$ of PIV recordings taken at this particular shock position. So far, the unsteadiness of SBLI has only been illustrated and discussed based on sample PIV shots taken for the turbulent case. The highly unsteady behaviour of separation has been observed in all four types of flow conditioning investigated but the frequency of separation varies with the type of flow control as will be discussed later. The chord-wise shock position has a further influence on size and location of the separation region. To assess latter variations, PIV samples are sorted after discrete passage shock positions. This conditional averaging is based on a few simplifying assumptions regarding the flow field which are discussed in the following.

\subsection{Discussion of uncertainties of flow assessment through conditional averaging}

To enable an assignment between shock position and separation, PIV samples are subdivided according to the normal shock position using bins of $1 \mathrm{~mm}$ spacing ( $1 \%$ of chord). The bin size is a compromise of having a sufficiently large sample count per bin for large shock excursions from the mean position, while keeping the spatial smoothing due to shock motion to a minimum. The position of the normal shock is automatically detected in each PIV sample as highlighted by the white vertical bar in the instantaneous velocity fields in Fig. 9. Occasional erroneous detections of the normal shock when the triple point is outside the boundaries of the imaged area lead instead to detections of the nearnormal shock and result in deviations of the order of spatial resolution of $1 \%$ of chord and thus have not been captured separately. The available number of PIV samples per shock position for a chord-wise resolution of $1 \mathrm{~mm}$ is provided in Fig. 12.

Furthermore, the conditional averaging procedure cannot distinguish between shocks traveling upstream from those traveling downstream. This could be of relevance considering that the relative velocity of the flow will be different depending of the shock traveling direction. To assess velocities of shock motion, the derivative of the time-resolved shock location is computed from time-resolved shadowgraphs in region A (see Figs. 5, 8). Table 2 shows, that the absolute mean velocities of shock motion are on the order of $1 \%$ and less compared to the pre-shock velocity for each of the investigated cases. Thus, the effect of different relative velocities is considered to be less significant because the velocities of the shock movement are very low compared to the in-flow velocity.

\subsection{Reduction of separation frequency and size by flow control}

The effectiveness of flow control with regard to suppression of BL separation is assessed on the basis of the number of vectors with negative velocity $n_{\mathrm{r}}$ in each individual PIV recording. Here, only negative $u$-components below a threshold of $u<-2 \varepsilon$ were considered as 'reverse flow' with $\varepsilon$ being the estimated signal peak detection accuracy for PIV (cf. Table 1). For the first 400 PIV samples of a recorded sequence, Fig. 11 shows the number of reverse flow vectors 
(a)
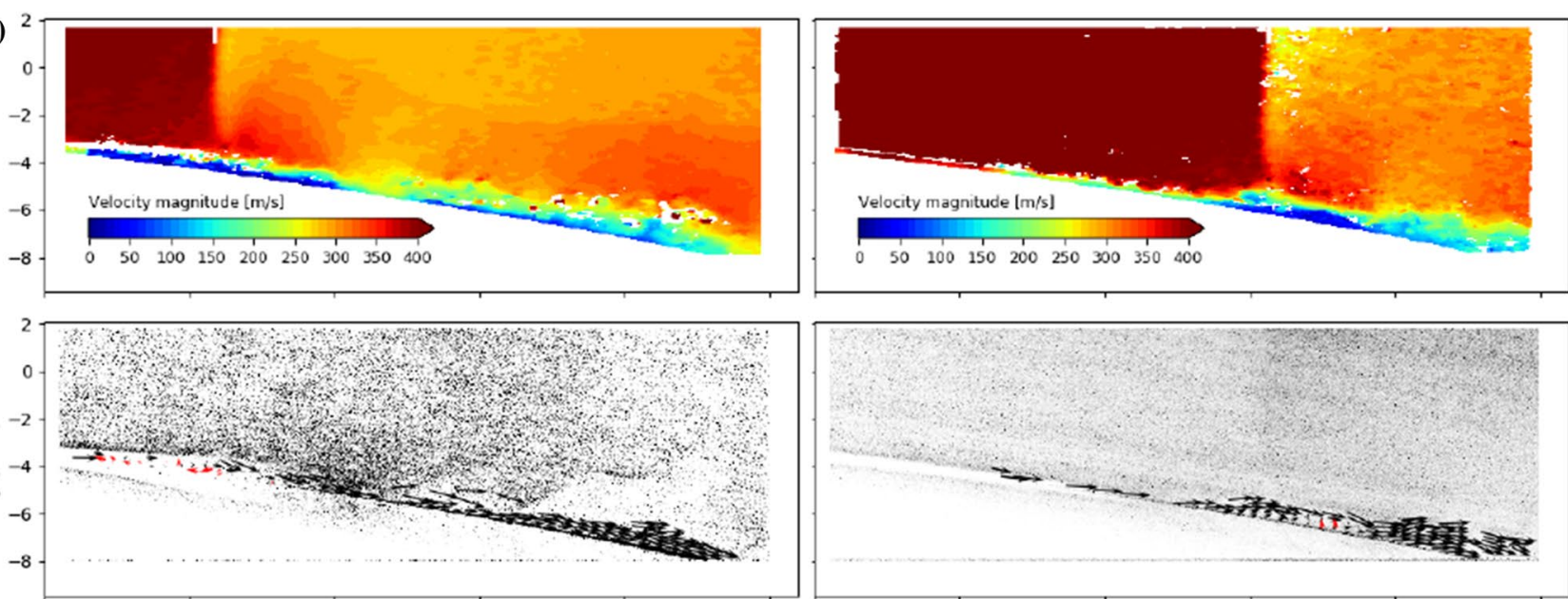

(b)
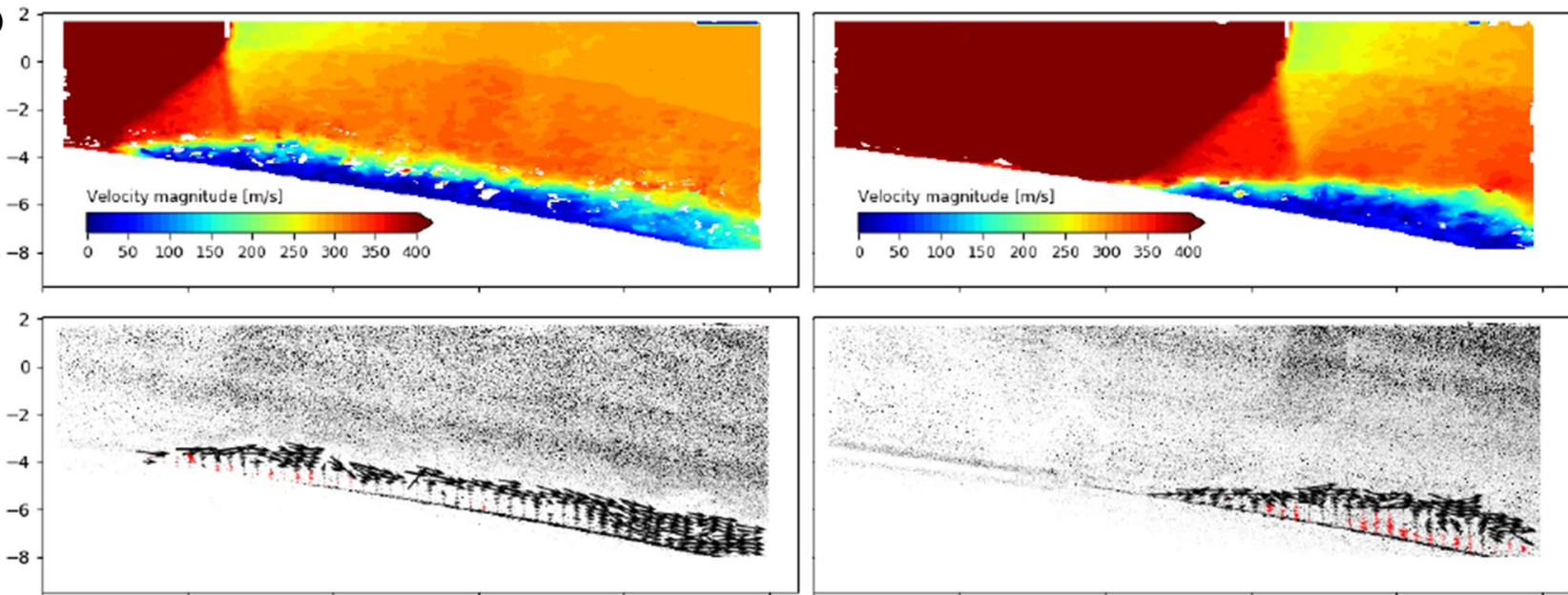

(c)
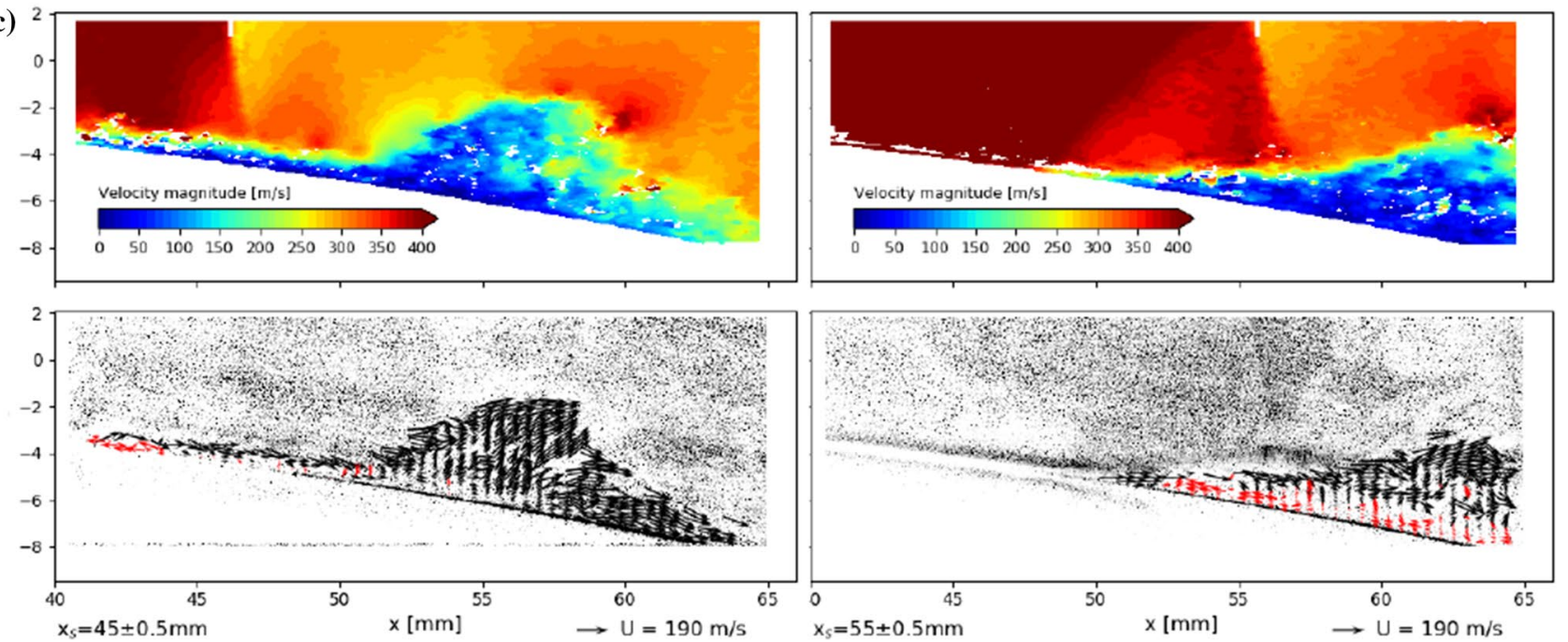

Fig. 9 Sample PIV shots from the turbulent test case with shocks further upstream (left) and downstream (right); vectors at $u>200 \mathrm{~m} / \mathrm{s}$ are clipped, vectors showing reverse flow are colored red; a nearly attached flow and normal passage shock; b flow separation with associated lambda pattern and slip line; $\mathbf{c}$ large flow separation including shape variation of the lambda pattern and occasional reverse flow 

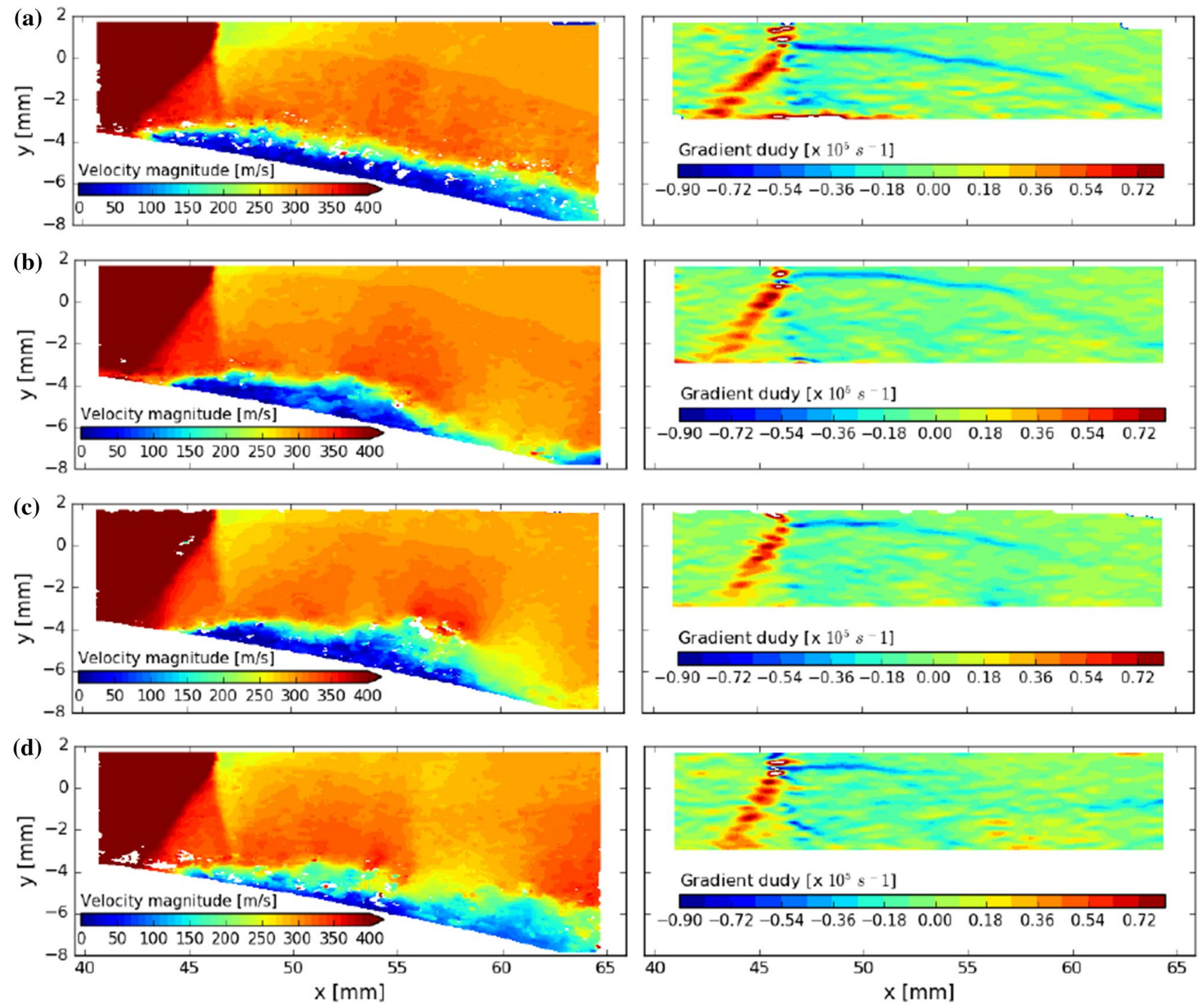

Fig. 10 Sample PIV shots of shock-induced flow separation at a similar shock position from the turbulent case; left: velocity magnitude; right: gradient $\mathrm{d} u / \mathrm{d} y$

related to the total number of visible vectors $n$ in the region of interest. The ratio $n_{\mathrm{r}} / n$ can be interpreted as an approximation of the area showing separated flow in each PIV sample. Under laminar conditions, large flow separations occur more frequently in comparison to the other three cases. At the same time, the AJVGs and the SRP exhibit larger regions

Table 2 Mean absolute velocity of the traveling shock wave in region A in Fig. 5 b; percentages are related to $M a_{1}=1.21$

\begin{tabular}{llll}
\hline Laminar case & Turbulent case & AJVGs & SRP \\
\hline $5.9 \pm 4.4 \mathrm{~m} / \mathrm{s}$ & $5.5 \pm 4.0 \mathrm{~m} / \mathrm{s}$ & $4.2 \pm 3.0 \mathrm{~m} / \mathrm{s}$ & $3.2 \pm 2.4 \mathrm{~m} / \mathrm{s}$ \\
$\left(1.4 \%\right.$ of $\left.u_{1}\right)$ & $\left(1.3 \%\right.$ of $\left.u_{1}\right)$ & $\left(1.0 \%\right.$ of $\left.u_{1}\right)$ & $\left(0.8 \%\right.$ of $\left.u_{1}\right)$ \\
\hline
\end{tabular}

of flow separation in sporadic PIV samples in comparison to the turbulent flow condition.

Based on this analysis, it is difficult to decide at which ratio $n_{\mathrm{r}} / n$ de facto BL separation occurs. In part, this is due to sporadic erroneous vectors present in areas of low particle image density in the separation region. Also, laser reflections from seeding deposits on the suction side contribute to sporadic outliers that influence the ratio. Choosing an arbitrary threshold of $n_{\mathrm{r}} / n>0.01$ (see dashed red line in Fig. 11) provides overall percentages across all PIV samples showing separation for each configuration, namely $93 \%$ for the laminar case, $37 \%$ for the turbulent case, $35 \%$ for AJVGs and 21\% for the roughness patch. These percentages clearly depend on the threshold but the ratio between these 
Fig. 11 Number of vectors showing reverse flow normalized with number of visible vectors in each of the first 400 PIV samples
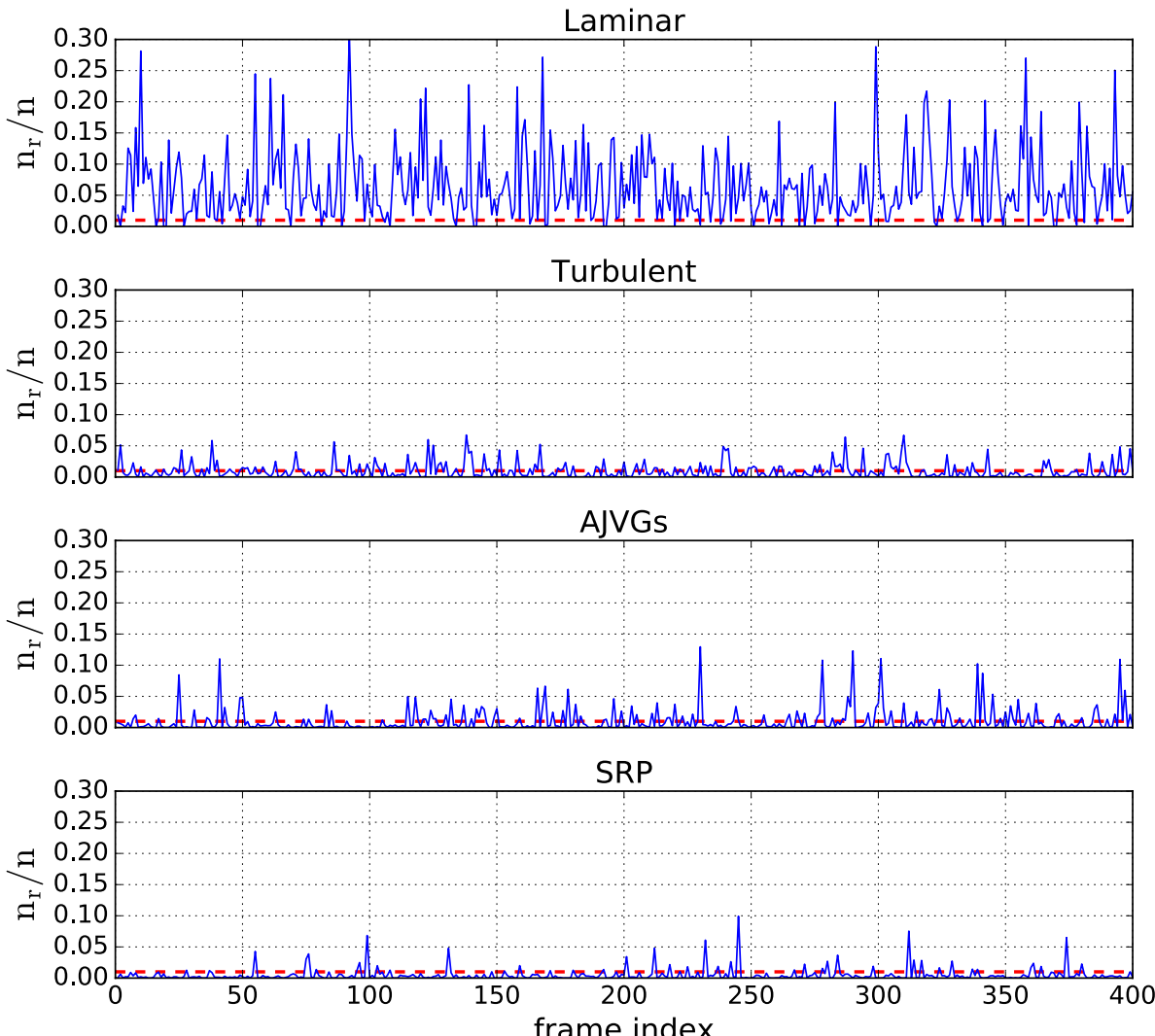

percentages can be seen as an rough estimate to compare the efficiency of flow control between these four configurations.

Based on the assumption already made above that separation occurs from an arbitrary threshold of $n_{\mathrm{r}} / n>0.01$, distributions can now be determined of the number of samples showing separation per shock position interval. These distributions are shown in Fig. 12 together with the number of PIV samples $N$ that falls in each shock position interval. It turns out that for AJVGs and for the SRP, separation occurs much more frequently downstream of the mean shock position. This effect is not so pronounced for the laminar and for the turbulent case.

To determine size and position of separation in relation to the shock position, the $x$ position of the first and last vector showing reverse flow has been extracted automatically from each individual PIV sample using the previously defined

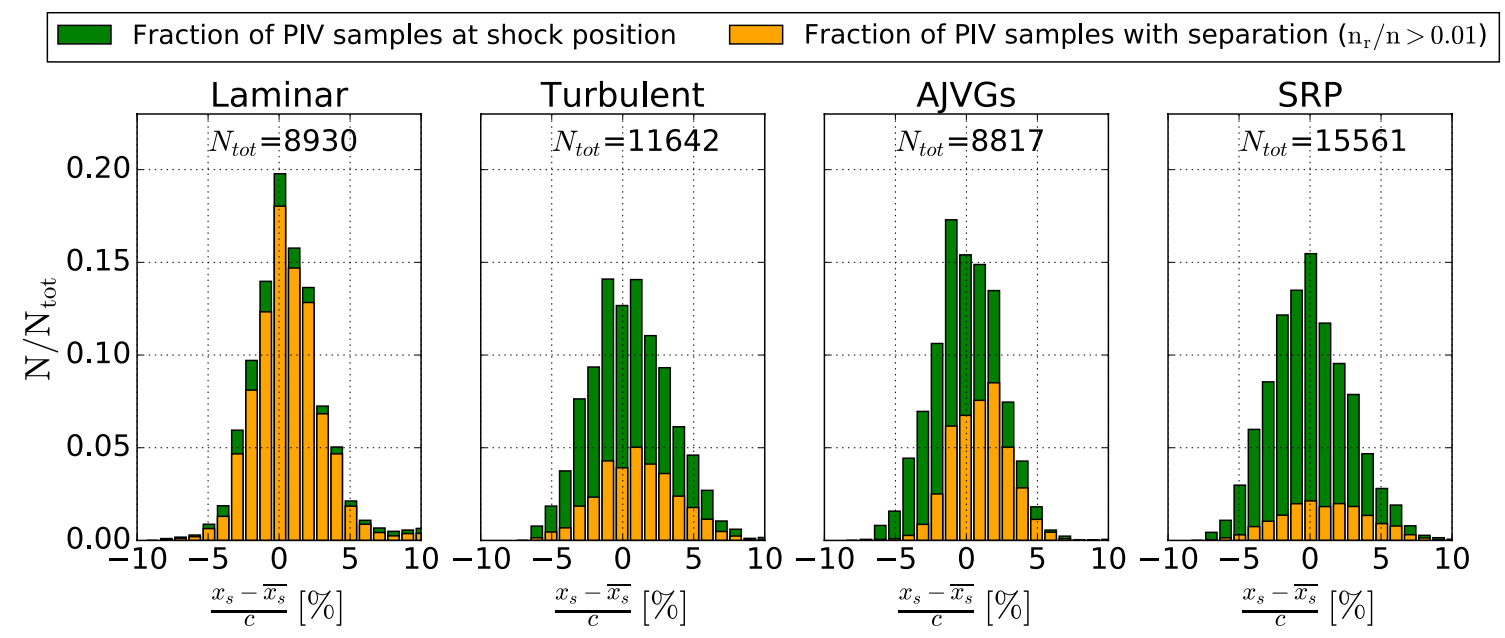

Fig. 12 Distribution of shock excursions from the mean position and corresponding fraction of samples with separation 
constraint $(u<-2 \varepsilon)$. Both vector instances are seen as instantaneous separation and reattachment points and are averaged within each shock position interval. Figure 13 relates the position and axial extend of the separation region with shock position, thus, determined for each configuration. In the laminar case, the detachment point is either upstream of the image boundaries or not detectable due to the very low seeding density of the laminar BL. In the laminar case, the reattachment points travel linearly with the shock position and exhibit a constant offset to the shock position of about $10 \mathrm{~mm}$ ( $10 \%$ of chord). For all other cases, the separation points are located in front of the normal shock. For the turbulent case and for the roughness patch, the axial extent of the separation region remains constant with respect to the shock position. A comparison of the spacing between separation and reattachment lines reveals an axial separation extent of approximately $16 \mathrm{~mm}$ (16\% of chord) under turbulent conditions which slightly increases to $18 \mathrm{~mm}$ if flow conditioning is applied using AJVGs or the SRP. The main difference for the last two cases is the different frequencies of separation over the total amount of PIV samples, that is about 3:2 between AJVGs and the roughness patch if a threshold of $n_{\mathrm{r}} / n>0.01$ is used.

\subsection{Boundary layer thickening and separation bubble size in the mean flow field}

The results provided in the following are based on sorting of PIV samples by discrete shock positions with a resolution of $1 \%$ of chord length, followed by averaging of the instantaneous velocity fields at each shock position interval. If reverse flow becomes visible in the conditionally averaged velocity field, the dividing streamline is computed by direct integration of axial velocities along the blade-normal axis. The position of the dividing stream line is where this integral of the velocity reaches zero (cf. Fitzgerald and Mueller 1990), indicating zero net mass flux with the external flow above. In this way, an estimate of the thickness of the separation bubble in the mean flow can be recovered. The resulting velocities fields based on conditional averaging at two shock positions of $4 \mathrm{~mm}$ spacing (4\% of chord) are shown in Fig. 14 left and right. The corresponding number of samples $N$ is specified in the plot.

In the case of low turbulence of the incoming flow (see Fig. 14a), the passage shock interacts with a laminar BL as shown using the LC visualizations. Near-wall velocities upstream of the SBLI could not be adequately evaluated because the particle density of the flow with laminar BL in most PIV snapshots is strongly reduced as only very few particles are carried from the outer flow into this area. Below and downstream of the SBLI, the particle image density is higher due to increased (turbulent) mixing with the outer flow and thus velocities inside of the separation bubble could be determined as well as the position of the dividing stream line. In particular for the laminar case, strong bubble separation occurs while the thickness of the bubble along $y$ increases approximately by a factor of 1.7 between both shock positions in Fig. 14a. The thickening of the separation bubble obviously also leads to an increase of the BL thickness which also varies with the shock position (cf. Table 3 ). Thus, at the rear shock position, stronger flow acceleration of the outer flow occurs compared to the outer flow at the upstream shock position (see Fig. 14a). At the downstream shock position, the reattachment point migrates significantly further downstream as already shown in Fig. 13. At the same time, the onset

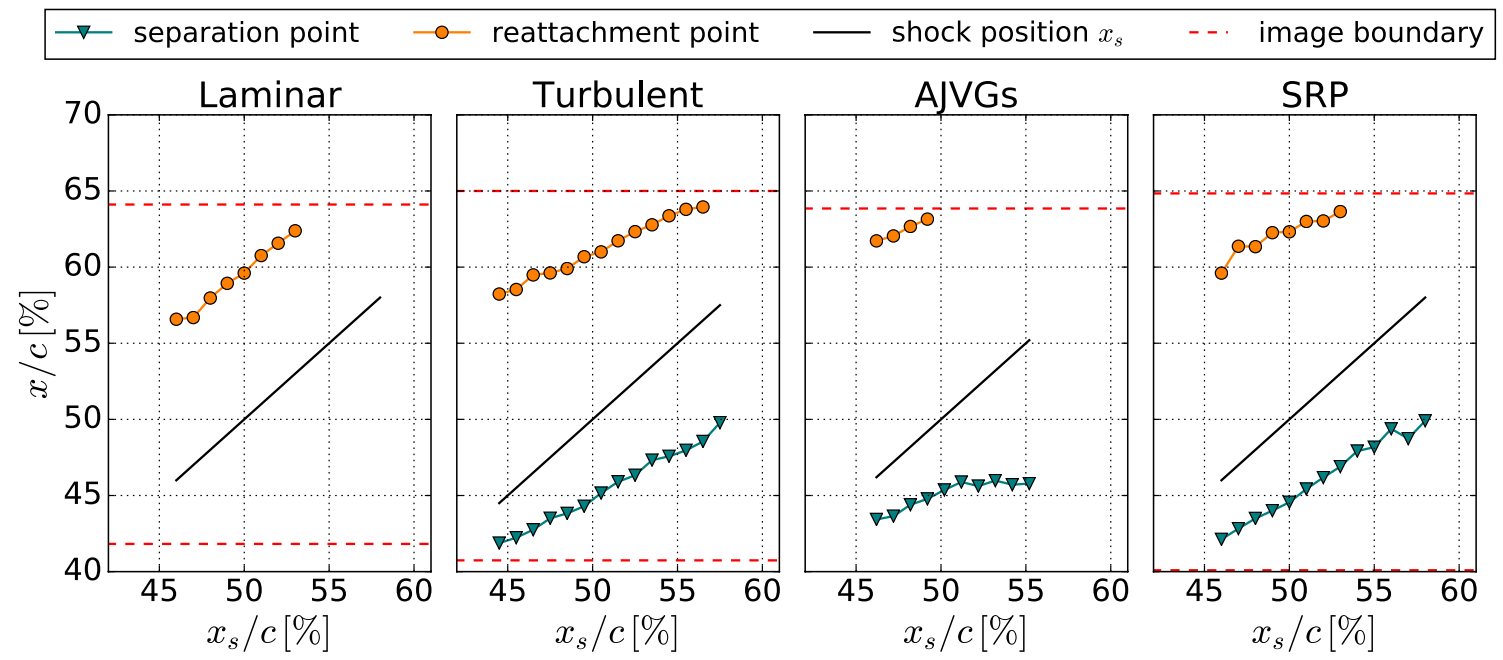

Fig. 13 Positions of separation and reattachment points with shock position based on statistics of the first and the last instance of reverse flow in each PIV sample 

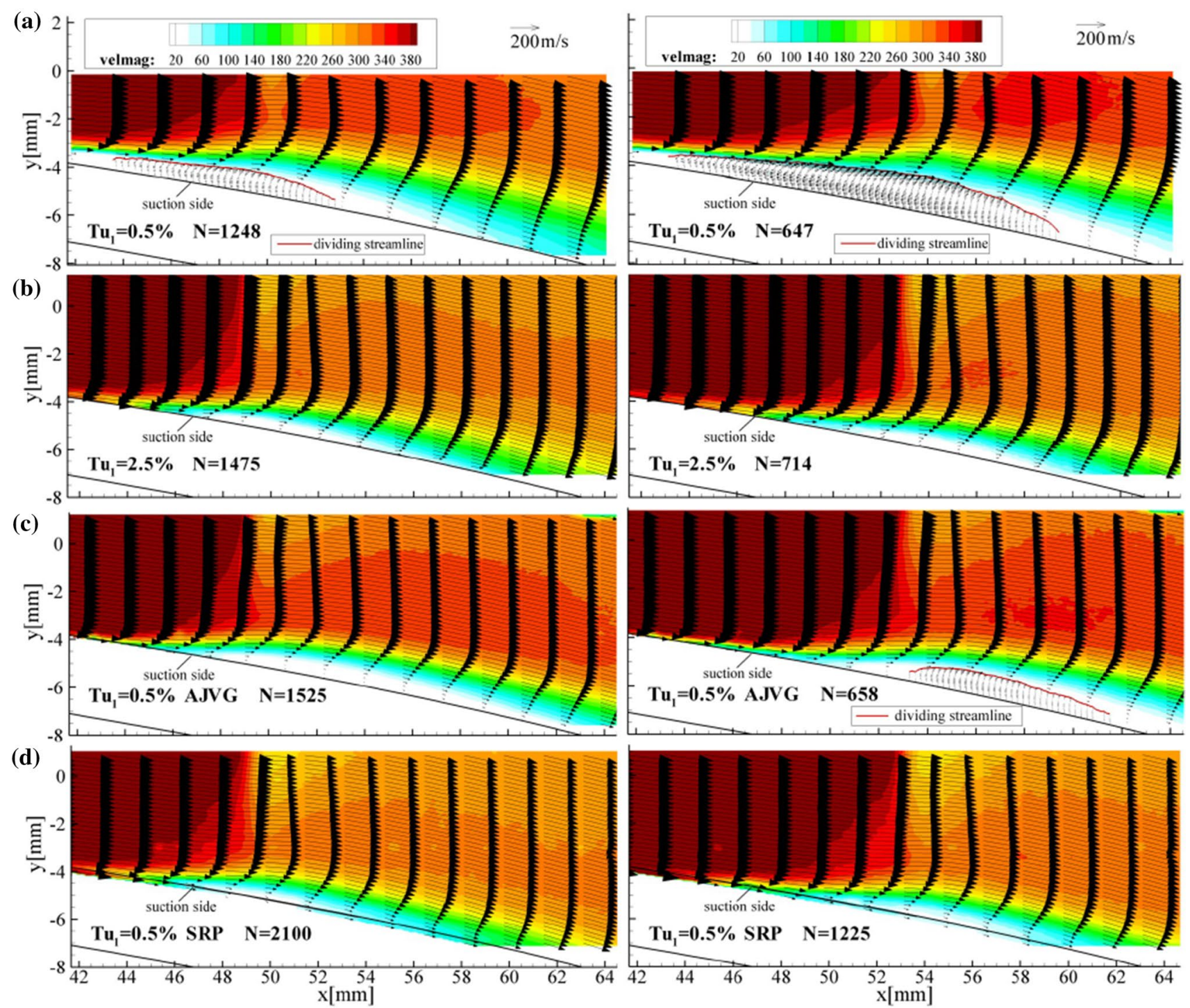

Fig. 14 Mean velocity at shock positions further upstream (left) and downstream (right) obtained by conditional averaging of PIV samples at $x_{\mathrm{s}}=49 \pm 0.5 \mathrm{~mm}$ and $x_{\mathrm{s}}=53 \pm 0.5 \mathrm{~mm}$; a laminar test case, $\mathbf{b}$ turbulent test case, $\mathbf{c}$ AJVGs, $\mathbf{d}$ SRP

Table 3 BL thickness $\delta_{99}$ downstream of the SBLI extracted from conditionally averaged velocities for two different shock positions shown in Fig. 14

\begin{tabular}{lll}
\hline$\delta_{99}(\mathrm{~mm})$ at $x=61 \mathrm{~mm}$ & $x_{\mathrm{s}}=49 \mathrm{~mm}$ & $x_{\mathrm{s}}=53 \mathrm{~mm}$ \\
$(x / c=0.61)$ & $x_{\mathrm{s}} / c=0.49$ & $x_{\mathrm{s}} / c=0.53$ \\
\hline Laminar case & 4.3 & 4.9 \\
Turbulent case & 3.6 & 3.7 \\
AJVGs & 3.1 & 3.8 \\
SRP & 2.9 & 2.9 \\
\hline
\end{tabular}

of separation is difficult to determine due to low seeding density, so that no conjectures can be made regarding the movement of the separation point with the shock position.
The attached animation of mean velocities at successive shock positions illustrates movement and thickening of the separation bubble depending on the shock position for the laminar case (see supplementary material 1).

For the turbulent case (cf. Fig. 14b), mean velocities at distinct shock positions do not exhibit bubble separation although evident from individual PIV shots in Fig. 9b, c. In the previous section, it was shown that regions with reverse flow occur only in about $37 \%$ of all PIV snapshots and that these regions are very small compared to reversed flow regions in the laminar case (cf. Fig. 11). Due to this, in the mean flow bubble separation is not evident. The BL thickness downstream of the SBLI is significantly lower than in the laminar case (cf. Table 3) and the increase of the BL thickness with the shock position is only marginal. 
For AJVGs (cf. Fig. 14c), a separation bubble appears only at the downstream shock position. This observation is consistent with the observation of more frequent separation events at shock positions downstream of the mean shock position (cf. Fig. 12). Due to the occurrence of bubble separation in the mean flow, the BL thickness downstream of the SBLI increases by more than $20 \%$ for AJVGs between both shock positions (cf. Table 3). The total axial length of the separation bubble at the downstream shock position is significantly smaller compared to laminar conditions, although the flow reattaches further downstream, as can also be seen in Fig. 13. For AJVGs, an animation is also available online showing the size variation of the separation bubble with the shock position (see supplementary material 2). In the animation, the separation bubble occurs first from $x_{\mathrm{s}} / c=51 \%$. From this position onward, the separation point remains fixed at a constant axial position (cf. Fig. 13), which leads to the presumption that the occurrence of a separation bubble in the mean flow seems to block the separation point at a fixed position even though the shock moves further downstream.

Due to the rare occurrence of separation for the roughness patch, the conditionally averaged flow field does not show any pronounced separation bubble (cf. Fig. 14d). The BL thickness downstream of the SBLI is lowest of all configurations and a variation of BL thickness with shock position could not be demonstrated (cf. Table 3). Based on this observation, a roughness patch with given dimensions, therefore, seems to be the most suitable approach toward reducing shock-induced flow separation under the given operating conditions.

\subsection{Spectral distribution of shock motion amplitudes}

Figure 15 shows the power spectral densities of passage shock motion obtained from high-speed shadowgraphs recorded at $20 \mathrm{kHz}$. For the laminar case and for AJVGs, two distinct peaks are noticeable at $f=1.14 \mathrm{kHz}$ with the first harmonic at $f=2.28 \mathrm{kHz}$ as well as a broader peak at $f=1.35 \mathrm{kHz}$. From literature, it is well known that periodic transonic shock oscillations (i.e. transonic buffet) can be triggered either by feedback loops in the separation bubble (Dussauge and Piponniau 2008) or by acoustic wave-propagation feedback from upstream propagating sound pressure waves (Lee 2001; Hartmann et al. 2013; Giannelis et al. 2017) whose source is assumed to originate from vortical flow structures passing over the trailing edge ('trailing edge noise'). Both test cases which show distinct harmonic components (laminar case and AJVGs), also exhibit bubble separation depending on the shock position (see Fig. 14). Possibly, this bubble separation may also support mechanisms that establish a feedback loop.

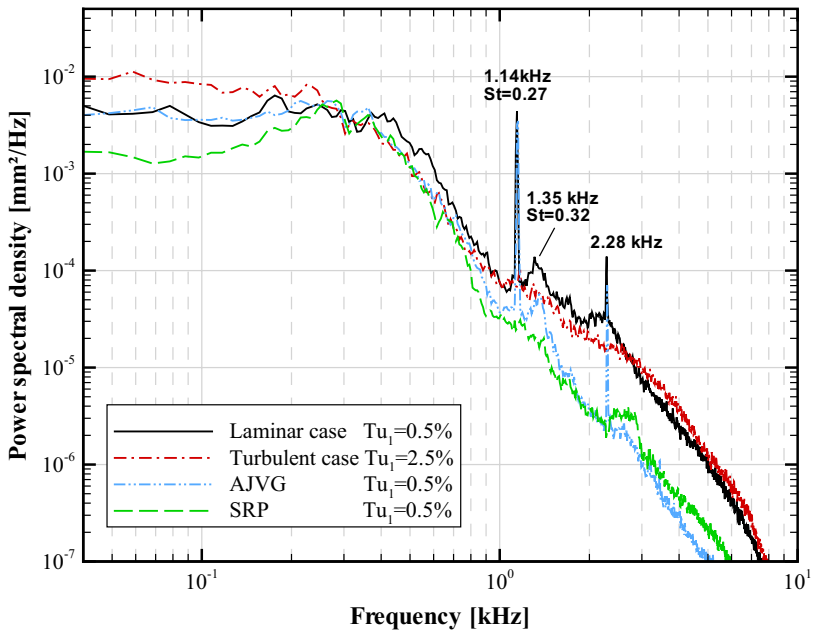

Fig. 15 Power spectral density of passage shock movement obtained from time-resolved shock shadowgraphs recorded in region $\mathrm{A}$ in Fig. 5b

Brion et al. (2019) investigated transonic buffet frequencies for a supercritical airfoil and found a dominant peak at a Strouhal number of $S t \approx 1.1$ for the laminar state of the incoming BL featuring bubble separation. In their paper, a dominant frequency also occurred for a turbulent state of the incoming $\mathrm{BL}$ at $\mathrm{St} \approx 0.07$. For the transonic cascade investigated here, the Strouhal number is $\mathrm{St} \approx 0.27(1.14 \mathrm{khz})$ and $\mathrm{St} \approx 0.32$ at the broader bump $(f=1.35 \mathrm{kHz})$. These Strouhal numbers are in between the results of Brion et al. which might be due to the different airfoil geometry or different average positions of the shock foot.

Apart from isolated peaks, the shock oscillation spectrum shown in Fig. 15 is dominated by a broadband spectrum. There are two frequency bands in which the power spectral densities of shock movement differs significantly between the test cases, namely a 'low-frequency band' ranging from 0.04 to $0.3 \mathrm{kHz}$ and a 'high-frequency band' ranging from 1 to $8 \mathrm{khz}$. Increasing the turbulence intensity of the incoming flow (turbulent case) is accompanied by the highest spectral densities of shock motion compared to other test cases. On the other hand, the case with the least pronounced flow separation (roughness patch) shows the lowest spectral densities of shock motion. For the laminar case and for AJVGs, the spectral densities in the low spectral range $(<0.3 \mathrm{kHz})$ are very similar and lie between the other two cases. In the high frequency range above $0.7 \mathrm{kHz}$, lower spectral densities are obtained for AJVGs and for the roughness patch. 


\subsection{Joined probability density distributions for shock position and positional blade displacements}

The shock location on the blade surface influences the surface pressure distribution and thus also the lift force that acts on the blade. This section investigates whether there is a correlation between the position of the normal shock and direction and strength of transverse blade displacement in region B (Fig. 5b). The latter type of displacement has also been used to correct the blade position in each PIV image.

A linear fit to positional blade displacements as a function of shock position (marked by the dashed line in Fig. 16) indicates a weak dependence of the direction of blade deflection from the shock position with cross-correlation coefficients of $R_{X Y}=0.09-0.12$, except for the laminar test case. The dependency is evident in that upward transverse blade displacements are more frequent when the shock is downstream from its mean position, while downward transverse blade displacements are more frequent at upstream positions of the shock.

Blade vibrations are minimal for the laminar case, in spite of the presence of strong bubble separation in this case. Among the remaining cases, the RMS of the positional blade displacements is lowest if a roughness patch is applied $\left(\sigma_{y}=0.067 \mathrm{~mm}\right)$. This coincides with both the lowest spectral densities of shock movement and the most distinctive suppression of flow separation (see Fig. 14) for the roughness patch. According to Fig. 16, the largest amplitudes of blade displacements occur for the test cases involving AJVGs or increased in-flow turbulence.

\section{Conclusions}

Two passive flow control devices, namely a surface roughness patch (SRP) and air-jet vortex generators (AJVGs), have been compared in regard to their suitability to reduce shock-induced flow separation and shock unsteadiness in a highly loaded compressor cascade at $M a_{1}=1.21$. In addition, two comparative cascade flows without transition control have been investigated that involve a low and a high degree of upstream turbulence (i.e., 'laminar' and 'turbulent' test cases). The size of the separation region was assessed based on large sets of statistically independent planar 2-C PIV samples measured near the suction side of the central blade of the cascade.

Individual PIV shots indicate strong variations in shape and position of the separation region as well as of the associated lambda shock pattern in all cases, even when there is no mean separation observed. The effectiveness of flow control with regard to reduction of BL separation is evaluated by comparing areas covered by reverse flow in each individual PIV sample. Under laminar conditions, large flow separations often occur, while the size of these areas is significantly reduced in the other three cases. The ratios of frequencies of BL separation between laminar case, turbulent case, AJVGs and SRP is approximately 1:0.4:0.38:0.23.

Conditional averaging upon different positions of the moving passage shock reveals strong bubble separation for the laminar case; whereas for AJVGs, a separation bubble only occurs when the shock is located at downstream positions. Bubble separation in the mean flow is not clearly evident from conditional averages if a roughness patch is
Fig. 16 Joined probability density distributions of transverse blade displacements $\Delta y$ versus shock position $x_{\mathrm{s}}$ in region $\mathrm{B}$ in Fig. $5 b$
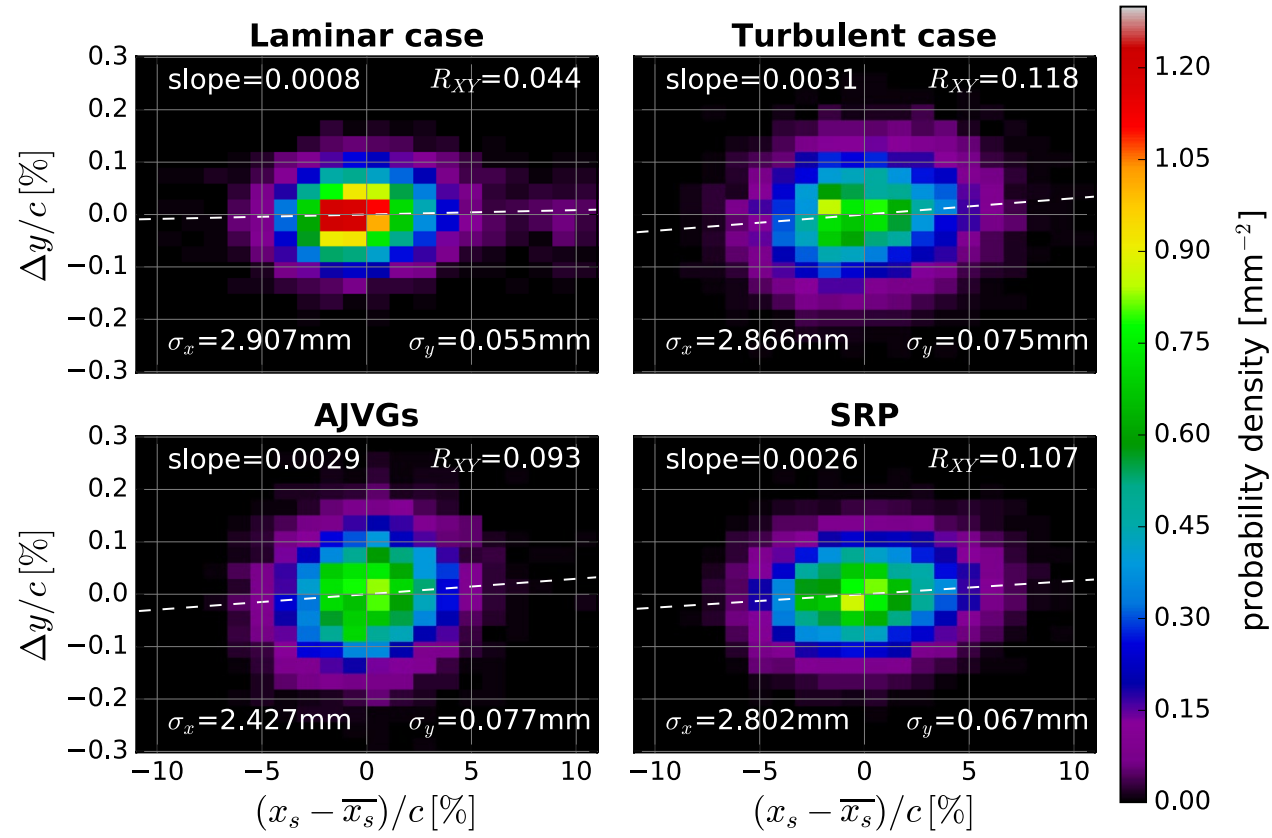
applied for transition control or when the turbulence level in the incoming BL is increased. Also the BL thickening downstream of the SBLI is lowest in the presence of the roughness patch. For laminar conditions and AJVGs, the BL thickness downstream of the SBLI increases significantly as the shock foot moves downstream.

Using time-resolved shadowgraph imaging, the position of the passage shock was tracked above the suction side of the central blade of the cascade. From these shadowgraphs, the spectral densities of the shock motion (i.e., squared amplitude of shock movement per frequency interval) could be recovered through Fourier analysis. The greatest damping of the shock movement in the low-frequency and high-frequency range could be achieved with the roughness patch, for which the separation region is also the least pronounced. Compared to the other cases, the turbulent case contains higher spectral densities in both the low-frequency and high-frequency range. By taking the conditionally averaged PIV results into consideration as well, this leads to the conclusion that a reduction in size of the separation region does not necessarily reduce the amplitudes of shock movement. However, only for laminar conditions and for AJVGs, a distinct peak at $\mathrm{St}=0.27$ becomes visible in the spectrum of shock motion which coincides with the occurrence of bubble separation in the mean flow. This relationship does not necessarily have to be causal and to prove this, time-resolved measurements would be necessary.

The joint probability density distributions between transverse positional blade displacements and amplitudes of shock movement reveal a weak dependency between the position of the near normal shock and the blade deflection. This can be observed in the way that upward transverse blade displacements are more frequent when the shock is downstream from its mean position while downward blade displacements are more frequent at upstream positions of the shock. The dependency is more pronounced for the turbulent test case and for AJVGs, which also show the highest amplitudes of blade vibrations compared to the other two test cases. With the exception of the laminar test case, the roughness patch imparted the lowest amplitudes of blade vibrations. It is clear that the blade response to shock oscillations strongly depends on the specific structural and damping characteristic of the airfoil. Further insight on this interdependence could be gained, for instance, by simultaneous time-resolved recording of shock movement and blade deflection.

Acknowledgements Part of the work presented herein is supported by the EU research project TFAST (Transition Location Effect on Shock Wave Boundary Layer Interaction, project no. 265455) of the 7th Framework Programme whose support is gratefully acknowledged. We thank our reviewers for their constructive remarks and detailed discussion on the content of the earlier version of the manuscript.

\section{References}

Babinsky H, Harvey JK (eds) (2011) Shock wave-boundary-layer interactions. Cambridge University Press, Cambridge

Brion V, Dandois J, Mayer R, Reijasse P, Lutz T, Jacquin L (2019) Laminar buffet and flow control. Proc Inst Mech Eng Part G J Aerosp Eng. https://doi.org/10.1177/0954410018824516

Délery JM, Bur RS (2000) The physics of shock wave/boundary layer interaction control: last lessons learned. In: European Congress on computational methods in applied sciences and engineering, ECCOMAS, Barcelona

Délery JM, Marvin J (1986) Shock-wave boundary layer interactions. AGARDograph 280, AGARD

Dolling DS (2001) Fifty years of shock-wave/boundary-layer interaction research: what next? AIAA J 39(8):1517-1531. https://doi. org/10.2514/2.1476

Dussauge JP, Piponniau S (2008) Shock/boundary-layer interactions: possible sources of unsteadiness. J Fluids Struct 24:1166-1175. https://doi.org/10.1016/j.jfluidstructs.2008.06.003

Fitzgerald EJ, Mueller TJ (1990) Measurements in a separation bubble on an airfoil using laser velocimetry. AIAA J 28(4):584592. https://doi.org/10.2514/3.10433

Flaszynski P, Doerffer P, Szwaba R, Kaczynski P, Piotrowicz M (2015) Shock wave boundary layer interaction on suction side of compressor profile in single passage test section. J Therm Sci 24(6):510-515. https://doi.org/10.1007/s11630-015-0816-9

Giannelis NF, Vio GA, Levinski O (2017) A review of recent developments in the understanding of transonic shock buffet. Prog Aerosp Sci 92:39-84. https://doi.org/10.1016/j.paero sci.2017.05.004. http://www.sciencedirect.com/science/artic le/pii/S0376042117300271

Giepman RHM, Schrijer FFJ, van Oudheusden BW (2015) High-resolution piv measurements of a transitional shock wave-boundary layer interaction. Exp Fluids 56(6):113. https://doi.org/10.1007/ s00348-015-1977-8

Hartmann A, Feldhusen A, Schröder W (2013) On the interaction of shock waves and sound waves in transonic buffet flow. Phys Fluids 25(2):026,101. https://doi.org/10.1063/1.4791603

Hartmann A, Klaas M, Schröder W (2012) Time-resolved stereo PIV measurements of shock-boundary layer interaction on a supercritical airfoil. Exp Fluids 52(3):591-604. https://doi. org/10.1007/s00348-011-1074-6

Hergt A, Grund S, Steinert W (2016) Webpage of the transonic cascade wind tunnel. https://www.dlr.de/at/en/desktopdefault.aspx/ tabid-1549

Hergt A, Klinner J, Grund S, Willert C, Steinert W, Beversdorff M (2019) On the importance of transition control at transonic compressor blades. In: Proceedings of ASME Turbo Expo Phoenix, Arizona, GT2019-90440

Hergt A, Klinner J, Wellner J, Willert C, Grund S, Steinert W, Beversdorff M (2018) The present challenge of transonic compressor blade design. In: Proceedings of ASME Turbo Expo Oslo, Norway, GT2018-75528

Jacquin L, Molton P, Deck S, Maury B, Soulevant D (2009) Experimental study of shock oscillation over a transonic supercritical profile. AIAA J 47(9):1985-1994. https://doi. org/10.2514/1.30190

Klinner J, Hergt A, Willert C (2014) Experimental investigation of the transonic flow around the leading edge of an eroded fan airfoil. Exp Fluids 55(9):1800. https://doi.org/10.1007/s0034 8-014-1800-y

Lee B (2001) Self-sustained shock oscillations on airfoils at transonic speeds. Prog Aerosp Sci 37(2):147-196. https://doi. org/10.1016/S0376-0421(01)00003-3 
Meyer R, Knobloch K, Linden J (2010) Hot-wire measurement in a high speed counter rotating turbo fan rig. In: Proceedings of ASME Turbo Expo Glasgow, UK, GT2010-22569

Raffel M, Willert C, Scarano F, Kähler C, Wereley S, Kompenhans J (2017) Particle image velocimetry. Exp Fluid Mech. https://doi. org/10.1007/978-3-319-68852-7

Ragni D, Schrijer F, van Oudheusden B, Scarano F (2011) Particle tracer response across shocks measured by PIV. Exp Fluids 50(1):53-64. https://doi.org/10.1007/s00348-010-0892-2

Sartor F, Losfeld G, Bur R (2012) PIV study on a shock-induced separation in a transonic flow. Exp Fluids 53(3):815-827. https://doi. org/10.1007/s00348-012-1330-4

Scarano F (2002) Iterative image deformation methods in PIV. Meas Sci Technol 13(1):R1. https://doi.org/10.1088/09570233/13/1/201. http://stacks.iop.org/0957-0233/13/i=1/a=201

Szwaba R (2011) Comparison of the influence of different air-jet vortex generators on the separation region. Aerosp Sci Technol 15(1):4552. https://doi.org/10.1016/j.ast.2010.06.001. http://www.scien cedirect.com/science/article/pii/S1270963810000714

Szwaba R (2013) Influence of air-jet vortex generator diameter on separation region. J Therm Sci 22(4):294-303. https://doi.org/10.1007/ s11630-013-0627-9
Szwaba R, Kaczynski P, Doerffer P (2019) Roughness effect on shock wave boundary layer interaction area in compressor fan blades passage. Aerosp Sci Technol 85:171-179. https://doi. org/10.1016/j.ast.2018.12.006. http://www.sciencedirect.com/ science/article/pii/S1270963818310794

Tejero F, Doerffer P, Szulc O (2015) Shock wave induced flow separation control by air-jet and rod vortex generators. TASK Q Sci Bull Acad Comput Centre Gdansk 19(2):167-180. http://yadda .icm.edu.pl/baztech/element/bwmeta1.element.baztech-389a6 977-77e0-4ca2-b6fb-39e13bd26223

Welch P (1967) The use of fast Fourier transform for the estimation of power spectra: a method based on time averaging over short, modified periodograms. IEEE Trans Audio Electroacoust 15(2):70-73. https://doi.org/10.1109/TAU.1967.1161901

Willert C, Mitchell D, Soria J (2012) An assessment of high-power light-emitting diodes for high frame rate schlieren imaging. Exp Fluids 53:413-421. https://doi.org/10.1007/s00348-012-1297-1

Publisher's Note Springer Nature remains neutral with regard to jurisdictional claims in published maps and institutional affiliations. 